Fact or artifact? Demand characteristics and participants' beliefs can moderate, but do not fully account for, the effects of facial feedback on emotional experience

\author{
Nicholas A. Coles ${ }^{1}$, Lowell Gaertner², Brooke Frohlich², Jeff T. Larsen², Dana M. \\ Basnight-Brown ${ }^{3}$ \\ ${ }^{1}$ Center for the Study of Language and Information, Stanford University, Stanford, USA \\ ${ }^{2}$ Department of Psychology, University of Tennessee, Knoxville, USA \\ ${ }^{3}$ United States International University-Africa, Nairobi, Kenya
}

\begin{abstract}
Author Note
Correspondence concerning this article should be addressed to Nicholas A. Coles. Email: ncoles@stanford.edu
\end{abstract}

All materials, code, and sharable data are available at https://osf.io/uf68k/. Study 1 video recordings are not available because participants did not agree to share these materials publicly. However, the coded data from the videos are available.

(CAmerican Psychological Association, 2022. This paper is not the copy of record and may not exactly replicate the authoritative document published in the Journal of Personality and Social Psychology. The final article is available, upon publication, at:

https://doi.org/10.1037/pspa0000316 


\begin{abstract}
The facial feedback hypothesis suggests that an individual's facial expressions can influence their emotional experience (e.g., that smiling can make one feel happier). However, a reoccurring concern is that supposed facial feedback effects are merely methodological artifacts. Six experiments conducted across 29 countries $(N=995)$ examined the extent to which the effects of posed facial expressions on emotion reports were moderated by (a) the hypothesis communicated to participants (i.e., demand characteristics) and (b) participants' beliefs about facial feedback effects. Results indicated that these methodological artifacts moderated, but did not fully account for, the effects of posed facial expressions on emotion reports. Even when participants were explicitly told or personally believed that facial poses do not influence emotions, they still exhibited facial feedback effects. These results indicate that facial feedback effects are not solely driven by demand or placebo effects.
\end{abstract}

Keywords: facial feedback hypothesis, demand characteristics, placebo effects, emotion, embodiment 


\section{Fact or artifact? Demand characteristics and participants' beliefs can moderate, but do not fully account for, the effects of facial feedback on emotional experience}

One of the fundamental goals of the social and affective sciences is to understand the nature of emotional experience. According to many modern theories, emotional experience is partially built off afferent signals from the peripheral nervous system (Cacioppo, Berntson, \& Klein, 1992; Damasio \& Carvalho, 2013; Laird \& Bresler, 1992; Levenson, Ekman, \& Friesen, 1990; Russell, 1980; Scherer \& Moors, 2019; Tomkins, 1962). If this is the case, it should be possible to modify people's emotional experiences by altering their peripheral nervous system activity. One of the most well-known demonstrations of this possibility comes from research examining the effects of sensorimotor facial feedback on emotional experience-i.e., the facial feedback hypothesis (Coles, Larsen, \& Lench, 2019; Coles et al., 2021; Izard, 1979; Strack, Martin, \& Stepper, 1988). For example, research suggests that posing happy expressions can cause people to feel happy and posing sad expressions can cause them to feel sad (Flack, 2006).

The facial feedback hypothesis suggest that researchers can test theories regarding the link between peripheral nervous system activity and emotion by manipulating facial feedback. This insight is particularly useful given that facial feedback can be precisely, ethically, and inexpensively manipulated (Rinn, 1984). Perhaps not surprisingly, then, researchers have examined the role of facial feedback in a variety of social and emotional processes. This includes emotion recognition (Marmolejo-Ramos et al., 2020; Niedenthal, Mermillod, Maringer, \& Hess, 2010; Wood, Rychlowska, Korb, \& Niedenthal, 2016), social perception (Niedenthal, Barsalou, Lawrence Winkielman, Krauth-Gruber, \& Ric, 2005), the experience of empathetic and vicarious emotions (Hatfield, Cacioppo, \& Rapson, 1992; Hoffman, 2001; Holland, Connell, \& Dziobek, 2020), attitude contagion (Skinner, Osnaya, Patel, \& Perry, 2020), the processing of emotional 
words and concepts (Neal \& Chartrand, 2011; Niedenthal, 2007; Niedenthal, Winkielman, Mondillon, \& Vermeulen, 2009; Winkielman, Coulson, \& Niedenthal, 2018), and decision making (Carpenter \& Niedenthal, 2020). Despite interesting advancements, though, a key issue remains unresolved: are facial feedback effects mere methodological artifacts?

\section{Explanations for facial feedback effects}

Many researchers posit that facial feedback exerts a genuine effect on emotional experience (see Figure 1), suggesting the effect is mediated by changes in:

1. Sensorimotor information, where facial feedback serves as a signal that provides information about an individual's emotional state (Allport, 1922; Izard, 1979, 2007; Laird, 1974; Schnall \& Laird, 2003; Tomkins, 1962).

2. Emotion-related cognitive processes, where facial feedback activates associated memories and/or impacts the processing of emotional information (Forster and Strack, 1996;

Niedenthal et al., 2005; Scherer, 2009; Schnall and Laird, 2003; Smith and Kirby, 2004;

Strack et al., 1988; Wood et al., 2016).

3. Motivational states, where facial feedback activates associated motivational states and/or behaviors (Coan, Allen, \& Harmon-Jones, 2001; E. Harmon-Jones, Gable, \& Price, 2011; Wansink, Zampollo, Camps, \& Shimizu, 2014).

4. Autonomic nervous system (ANS) activity, where facial feedback activates associated patterns of autonomic nervous system activity (Ekman, Levenson, \& Friesen, 1983; Kraft \& Pressman, 2012; Levenson, Carstensen, Friesen, \& Ekman, 1991; Levenson et al., 1990; Levenson, Ekman, Heider, \& Friesen, 1992).

Alternatively, other researchers posit that the effect of facial feedback on emotional reports is a methodological artifact (see Figure 1) —one driven by demand characteristics (Buck, 
1980; Schimmack \& Chen, 2017) and/or participants' beliefs about facial feedback (Arnone et al., 2021; Coles \& Larsen, 2021; Coles, Larsen, Kuribayashi, \& Kuelz, 2019; Rudorfer, 2018).

As shown in Figure 1, we define demand characteristics as cues that make participants aware of the experimenter's hypothesis (but see Corneille and Lush, 2021 for a review of the phrase's polysemy). We refer to the effect of demand characteristics on participants' responses as demand effects. We posit that demand effects impact participants' emotion reports—not their actual experience of emotion - and assume that this is driven by participants' desire to help the experimenter confirm their hypothesis. This characterization of demand effects is often called "good subject effects" (Orne, 1959, 1962; for a review, see Strohmetz, 2008), although we review other types of demand effects in the General Discussion.

Also shown in Figure 1 is our assumption that participants' beliefs lead to placebo effects on their experience of emotion. Although often considered distinct, demand effects and placebo effects are also related. They are distinct because researchers theorize that they have different underlying mechanisms: motivation to help the experimenter (demand effect) and belief in the hypothesized effect (placebo effect). Furthermore, demand effects impact participants' emotion reports (e.g., exaggerating happiness reports), whereas placebo effects impact participants' experience of emotion (e.g., feeling subjectively happier). Demand and placebo effects are related, however, because demand characteristics can trigger both demand effects and placebo effects (see Figure 1; Corneille \& Lush, 2021). For example, imagine a researcher tells a participant that they hypothesize smiling increases happiness (i.e., there are demand characteristics). The participant might want to help confirm the hypothesis and change their emotion reports accordingly (i.e., a demand effect). Alternatively, the participant may believe the hypothesis and experience placebo-induced changes in their emotional states. 


\section{Figure 1}

Facial feedback as a genuine effect (dotted lines) vs. methodological artifact.

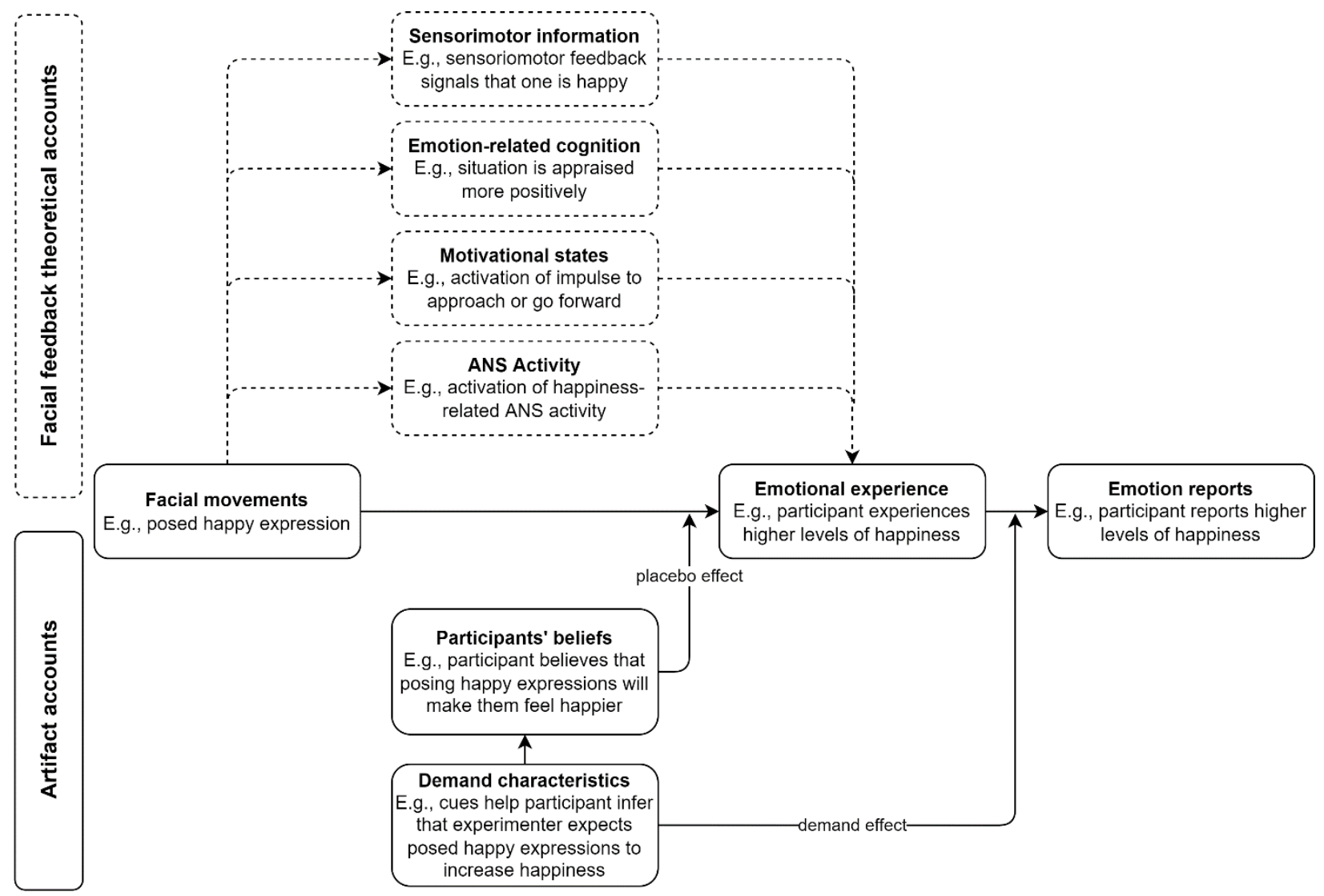




\section{Concerns about demand and placebo effects in the facial feedback literature}

Demand and placebo effects are particularly concerning in the facial feedback literature because a recent meta-analysis indicated that $83 \%$ of facial feedback studies have featured overt facial pose manipulations (Coles, Larsen, \& Lench, 2019). For example, Schnall and Laird (2003) instructed participants to "produce the facial expression that you would have when you were extremely happy" (p. 790). These overt facial pose manipulations may lead to demand or placebo effects by causing participants to (a) guess the experimenter's hypothesis and adjust their ratings accordingly, and/or (b) form expectations about the effects of facial poses on emotion that subsequently influence their emotional experience. The remaining $17 \%$ of studies featured unobtrusive facial-pose manipulations in an effort to conceal the experimenter's hypothesis. For example, in a study ostensibly about psychomotor coordination, Strack, Martin, and Stepper (1988) had participants view humorous cartoons while holding a pen in their mouth in a manner that either induced or inhibited smiling. Similarly, in a study ostensibly about facial muscle reaction times, Dimberg and Söderkvist (2011) asked participants to react to nature scenes by contracting their zygomaticus major (smiling) or corrugator supercilii (scowling) facial muscles. Consistent with the facial feedback hypothesis, participants in both studies reported more positive reactions to the stimuli after smiling despite apparently being unaware of the purpose of the facial feedback manipulation.

Studies that have featured unobtrusive facial pose manipulations, however, have yielded mixed results. For example, a collaborative effort involving 17 teams failed to replicate the penin-mouth effect (Wagenmakers et al., 2016; but see Marsh et al., 2018 and Noah et al., 2018). Likewise, in a conceptual replication of Dimberg and Söderkvist (2011), Söderkvist, Ohlén, and Dimberg (2018) did not find that participants reported more positive reactions to pleasant stimuli 
after completing an unobtrusive smiling task. More broadly, Coles et al.'s (2019) meta-analysis revealed a reliable effect of facial feedback on self-reported emotional experience among studies that used overt facial manipulations, but not among studies that used unobtrusive facial manipulations. Similarly, an adversarial collaboration involving 26 teams spanning 19 countries consistently found that overt facial feedback manipulations increased happiness, but they did not consistently find evidence of such effects when using an unobtrusive pen-in-mouth procedure (Coles et al., 2021). This discrepancy between results obtained via overt vs. unobtrusive facial feedback manipulations is consistent with long-standing concerns that facial feedback effects are nothing more than methodological artifacts.

To summarize, concerns about demand and placebo effects in the facial feedback literature persist. Before investing resources into studying the theoretically interesting mechanisms that may underlie facial feedback effects, we argue that researchers should first thoroughly examine whether such effects are driven by methodological artifacts. We do so in the present work, testing the impact of (a) demand characteristics in Studies 1-3 and (b) participants' beliefs about facial feedback in Studies 4-6. 


\section{Overview of Studies 1-3}

We designed Studies 1-3 to examine the extent to which facial feedback effects are driven by demand characteristics. To test facial feedback effects, we had participants self-report their emotions after posing happy, angry, and neutral expressions. We chose happy expressions because Coles et al.'s (2019) meta-analysis indicated that it is the most frequently manipulated expression in the facial feedback literature. We chose angry expressions as a negative-valenced counterpart because the same meta-analysis indicated that posing angry expressions had a larger overall effect on emotional experience $(d=0.53)$ than posing expressions of other negative emotions (e.g., sadness: $d=0.30)$. Furthermore, prior work (e.g., Dimberg \& Söderkvist, 2011) and our own pilot work (Coles, 2020) indicated that relatively simple instructions could be used to have participants pose the critical components of happy expressions (i.e., smiling) and angry expressions (i.e., scowling).

To examine the role of demand characteristics, we used an experimental approach originally proposed by Orne (1959). Whereas previous facial feedback researchers have attempted to limit demand characteristics (e.g., R. J. Larsen, Kasimatis, \& Frey, 1992; Strack et al., 1988), we directly manipulated them by communicating different hypotheses to different participants. (For similar approaches, see de Quidt, Haushofer, \& Roth, 2017; Gustafson \& Orne, 1965; Kersbergen et al., 2019; J. T. Larsen \& McGraw, 2011; Mummolo \& Peterson, 2019; Nichols \& Maner, 2008; Orne, 1959; Roche, Forsyth, \& Maher, 2007; Sigall, Aronson, \& Hoose, 1970; Veitch, Gifford, \& Hine, 1991; Young, Adelstein, \& Ellis, 2006). Before participants engaged in facial pose tasks, we randomly assigned them to one of three conditions. In the positive-hypothesis condition, we told participants we sought to demonstrate that their facial poses will influence their emotions. In the nil-hypothesis condition, we told participants that we 
sought to demonstrate that their facial poses will not influence their emotions (i.e., that we hypothesized null effects). In the no-hypothesis condition, we did not provide participants with information about the goal of the experiment.

There are three reasons we manipulated demand characteristics in Studies 1-3. First, amid ongoing debates about the replicability of studies that have featured unobtrusive facial feedback manipulations, we sought a potentially more robust approach for studying the effects of demand characteristics. Second, manipulating demand characteristics allowed us to better assess causal relationships between demand characteristics and facial feedback effects (Weber \& Cook, 1972). Consistent with concerns about demand characteristics, correlational research indicates that participants who report being aware of the purpose of facial feedback studies show larger facial feedback effects (Coles et al., 2021; Kaiser \& Davey, 2017). However, the reverse direction of causation is also plausible; participants who experience stronger facial feedback effects may subsequently be better able to infer the purpose of the study. Furthermore, the association between facial feedback effects and awareness of the purpose of the study may be spurious. For example, higher levels of focus might independently cause participants to experience stronger facial feedback effects and better infer the purpose of a study.

Third, manipulating demand characteristics allowed us to conduct what Mayo (1991) terms a severe test of the facial feedback hypothesis. In Mayo's framework, the strength of evidence for a hypothesis is a function of test severity: the probability of falsely yielding hypothesis-consistent results. For example, compared to non-blinded trials, double-blind trials of an ineffective drug are less likely to yield false positives because they limit the role of potential methodological artifacts like demand characteristics. Consequently, double-blinded medical trials provide more severe tests of new interventions than non-blinded medical trials. Similarly, if 
the facial feedback hypothesis is false, studies in which demand characteristics work against the facial feedback hypothesis are less likely to falsely yield facial feedback effects than studies in which demand characteristics are merely minimized. Consequently, the nil-hypothesis condition in our three studies (i.e., the one in which we told participants the goal is to prove that facial feedback does not impact emotion) provides a more severe test than previous studies that used unobtrusive facial feedback manipulations to minimize the role of demand characteristics.

\section{Study 1}

Researchers generally posit that demand characteristics cause participants to respond consistently with what they perceive to be the experimenter's hypothesis (Orne, 1959, 1962; for a review, see Strohmetz, 2008). Consequently, we predicted that facial feedback effects would be largest in the positive-hypothesis condition and smallest in the nil-hypothesis condition. We further assumed that facial feedback effects are fully driven by demand characteristics. Thus, we predicted that facial feedback effects would not only be smallest, but more specifically absent, in the nil-hypothesis condition.

\section{Method}

\section{Participants and design}

We used a 3 (Pose: happy, angry, neutral) x 2 (Block: first or second) x 3 (Demand: positive-hypothesis, no-hypothesis, nil-hypothesis) mixed design, with Demand manipulated between-subjects. We determined sample size with a power simulation that assumed the effects of posed expressions on emotion would be large in the positive-hypothesis condition and nonexistent in the nil-hypothesis condition. Based on this power simulation, we planned to recruit at least 250 participants to achieve (a) $\approx 99 \%$ power to detect the hypothesized Pose by Demand 
interactions, and (b) $>70 \%$ power to detect our hypothesized Pose effects within the positivehypothesis and no-hypothesis conditions (for details, see https://osf.io/uf68k/).

Two hundred and fifty-one psychology students from the University of Tennessee (a large southeastern university in the United States) completed the experiment in exchange for partial course credit between October $29^{\text {th }}, 2019$, and March $10^{\text {th }}, 2020$. Per our pre-registered analysis plan, we excluded one participant who did not respond to the emotion self-report measures. This left us with data from 250 participants $\left(67 \%\right.$ women, $33 \%$ men, $M_{\text {age }}=18.94$, $S D_{\text {age }}=2.37$; for all available participant pool ethnicity data, see the Supplemental Materials).

\section{Procedure}

Participants arrived at the laboratory one at a time and were told by an experimenter that (a) the study examined how muscle movements and fluctuations in concentration/mood influence galvanic skin response and (b) they were assigned to move the muscles in their face. We provided this cover story to ensure that participants in all conditions were aware of our interest in facial movements. Next, an experimenter attached two electrodes to participants' left hand. These electrodes were connected to a visibly active Biopac MP36, although no physiological recordings were actually taken. An experimenter then informed participants that they would complete six timed facial movements and answer questions about their concentration and mood.

Participants were randomly assigned to one of three Demand conditions using the random assignment feature in Qualtrics. ${ }^{1}$ Participants in the no-hypothesis condition $(n=77)$ began the facial expression poses without further instruction. Participants in the positive-hypothesis $(n=$

${ }^{1}$ In Studies 1-3, participants were assigned to demand condition using the random assignment feature in Qualtrics. However, quota counts were not used to force an equal distribution of participants across conditions. Consequently, sample sizes differ slightly across conditions. 
86) and nil-hypothesis conditions $(n=87)$ received the following message from the experimenter (nil-hypothesis message in brackets):

"Researchers know that facial expressions influence skin responses. However, they are not sure why. Some researchers think that this happens because posing a facial expression of emotion causes you to feel that emotion. That is, smiling may make you happier and scowling may make you angrier. We believe this is $[n o t]$ true. We are running this study to prove our hypothesis that posing facial expressions [does not $]$ cause you to experience that emotion."

If the participant indicated that they did not understand the above message, an experimenter repeated the information with a visual aid (Figure S1 in Supplemental Materials). Experimenters then left participants to complete the experiment alone, although participants were covertly recorded using a hidden webcam.

Participants first completed two blocks of poses, each which featured the same instructions to pose happy, angry, and neutral facial expressions in random order. For happy poses, participants were instructed to move the corner of their lips towards their ears, elevating their cheeks (Dimberg \& Söderkvist, 2011). For angry poses, participants were instructed to move their eyebrows down towards their nose (Dimberg \& Söderkvist, 2011). For neutral poses, participants were instructed to maintain a blank expression. Participants were asked to hold each pose for 5 seconds in conjunction with an on-screen timer. After each pose, participants completed a modified Discrete Emotions Questionnaire (C. Harmon-Jones, Bastian, \& HarmonJones, 2016), which asked them to report the extent ( $0=$ "not at all" to $6=$ "an extreme amount") to which they experienced happiness (three averaged items: happiness, satisfaction, and enjoyment; overall $a=.88$ ), anger (three averaged items: irritation, aggravation, and annoyance; 
overall $\mathrm{a}=.85$ ), and fear (alarmed, scared, and fear) during the preceding posing trial. ${ }^{2}$

Participants also reported how difficult they found the posing task and how much they concentrated. Exploratory analyses related to self-reported difficulty and concentration did not change our conclusions and are not discussed further. After the two blocks of poses, participants completed the Multidimensional Assessment of Interoceptive Awareness (Mehling et al., 2012). We did not pre-register any hypotheses for this measure and do not report analyses here.

After completing the main portion of the experiment, participants pressed a button that notified an experimenter to return and begin a funnel debriefing. In the funnel debriefing, an experimenter asked participants questions about their beliefs about the purpose of the study. Based on these funnel debriefings, the experimenter provided ratings of the degree to which the participant was aware we were testing facial feedback effects $(0=$ "not at all aware" to $4=$ "completely aware"). We included this measure to check that participants were more aware we were testing facial feedback effects in the positive-hypothesis and nil-hypothesis conditions (vs. the no-hypothesis condition). The University of Tennessee Institutional Review Board approved the experimental protocol.

\section{Results}

\section{Awareness that Experiment Tested Facial Feedback Effects}

To examine the efficacy of the demand characteristics manipulation, we tested whether participants were rated as more aware that we were testing facial feedback effects in the positive-

${ }^{2}$ The original Discrete Emotions Questionnaire contains four items measuring happiness, anger, fear, disgust, anxiety, sadness, desire, and relaxation. Given our interest in happiness and anger, we excluded all other subscales besides fear, which was used as filler items. To shorten the subscales, we used three (as opposed to four) items per subscale. The original anger subscale contains the following items: anger, rage, mad, and pissed off. We felt that these only captured high-intensity anger and instead used irritation, aggravation, and annoyance. Cronbach alphas were calculated separately for each pose and block combination and then averaged. 
and nil-hypothesis conditions (vs. the no-hypothesis condition). Caution is warranted because experimenters were aware of participants' condition, which could have influenced their behavior and interpretation of participants' responses (Rosenthal \& Fode, 1963). Nonetheless, a one-way ANOVA confirmed that awareness ratings varied across Demand conditions, $F(2,247)=31.07$, $p<.001$. Participants were less aware we were testing facial feedback effects in the nohypothesis condition $(M=1.39, S D=1.23)$ than in the positive-hypothesis $(M=2.93, S D=$ $1.22), t(247)=7.66, p<.001, M_{\text {diff }} 95 \%$ CI $[1.14,1.94]$, and nil-hypothesis conditions $(M=2.52$, $S D=1.39), t(247)=5.62, p<.001, M_{\text {diff }} 95 \%$ CI $[0.73,1.52]$. Less critically, participants were slightly more aware we were testing facial feedback effects in the positive-hypothesis than nilhypothesis condition, $t(247)=2.12, p=.04, M_{\text {diff }} 95 \% \mathrm{CI}[0.03,0.80]$.

\section{Adherence to Facial Posing Task Instructions}

To probe the efficacy of the facial pose manipulation, we examined the extent to which participants posed the target facial expressions during the posing trials. ${ }^{3}$ For the 209 participants who (a) consented for their videos to be analyzed, and (b) had usable video footage, we processed video recordings of their posing trials through Noldus FaceReader 6.0 (Lewinski, den Uyl, \& Butler, 2014). This program computed moment-to-moment scores of expressed happiness and anger on a continuous 0.0 to 1.0 scale. We subsequently modeled participants' expressed happiness and anger scores using linear mixed-effects regressions with Pose entered as a fixedeffect factor and random-intercepts for participants. The $F$-values we report are from ANOVA tables with Satterthwaite degrees of freedom.

${ }^{3}$ Pilot work indicated that participants complete the facial expression posing tasks well (Coles, 2020), so we did not intend to re-examine this assumption in Study 1. Nevertheless, analysis of the video recordings provides further evidence of the efficacy of the facial expression pose manipulation. We thank an anonymous reviewer for recommending the analysis. 
Results confirmed that participants successfully completed the facial expression posing tasks. Participants displayed more happiness during the happy $(M=0.37, S D=0.34)$ vs. neutral $(M=0.01, S D=0.03), t(1043)=28.70, p<.001$, and angry $(M=0.01, S D=0.04), t(1043)=$ $28.67, p<.001$, posing trials. Participants also displayed more anger during the angry $(M=0.24$, $S D=0.29)$ vs. neutral $(M=0.07, S D=0.16), t(1043)=15.34, p<.001$, and happy $(M=0.03$, $S D=0.10), t(1043)=18.49, p<.001$, posing trials. Less critically, we did not observe that participants displayed different levels of happiness in the neutral vs angry posing trials, $t(1043)=$ $-0.03, p=.98$. Also less critically, we observed that participants displayed lower levels of anger in the happy vs neutral posing trials, $t(1043)=-3.16, p=.002$.

\section{Self-Reported Emotional Experience}

We modeled emotion reports using linear mixed-effects regression with Pose, Block, and Demand entered as effects-coded, fixed-effect factors, all higher-order interactions, randomintercepts for participants, and Type III Sums of Squares. ${ }^{4} F$-values are from ANOVA tables with Satterthwaite degrees of freedom. The effect of interest was the Pose by Demand interaction, but we report results from the full model in the Supplemental Materials. We used Wilcoxon tests for all pairwise comparison tests, and we additionally report model-estimated marginal means, pairwise comparison confidence intervals, and effect sizes in the Supplemental Materials.

${ }^{4}$ Participants' emotion reports in our studies were generally zero-inflated, with a large proportion of participants indicating that they experienced no emotion or very little emotion. Many researchers argue that linear mixed-effects models are robust to such violations of distributional assumptions (Schielzeth et al., 2020). Nevertheless, we observed similar results when we modeled emotion reports using logistic mixed-effects regressions (with emotion reports dichotomized as zero or non-zero). 
Self-Reported Happiness. Results indicated that there was a significant Pose by Demand interaction, $F(4,1235)=24.60, p<.001$. As depicted in the top panel of Figure 2, the simple effect of Pose on self-reported happiness was largest in the positive-hypothesis condition, $F(2$, $1235)=191.47, p<.001$, second largest in the no-hypothesis condition, $F(2,1235)=85.53, p<$ .001 , and smallest in the nil-hypothesis condition, $F(2,1235)=16.79, p<.001$. In all Demand conditions, participants reported more happiness after posing happy vs. neutral or angry expressions (Figure 2). In other words, contrary to our initial prediction, participants exhibited significant facial feedback effects even when we explicitly informed them that the purpose of the study was to demonstrate that facial poses do not impact emotions. These results indicate that demand characteristics moderated, but did not fully account for, the effects of posed expressions on happiness.

Self-Reported Anger. Once again, results indicated that there was a significant Pose by Demand interaction, $F(4,1235)=17.55, p<.001$. As depicted in the bottom panel of Figure 2, the simple effect of Pose on self-reported anger was largest in the positive-hypothesis condition, $F(2,1235)=116.86, p<.001$, second largest in the no-hypothesis condition, $F(2,1235)=54.42$, $p<.001$, and smallest in the nil-hypothesis condition $F(2,1235)=8.89, p<.001$. In all Demand conditions, participants reported more anger after posing angry vs. neutral or happy expressions (Figure 2). Similar to the findings with happiness, these results indicate that demand characteristics moderated, but did not fully account for, the effects of posed expressions on anger.

\section{Discussion}

Study 1 provides the first experimental evidence that facial feedback effects are moderated by participants' knowledge of the study hypothesis (i.e., demand characteristics). 
However, contrary to our initial predictions, posed happy and angry facial expressions influenced emotional experience even when we explicitly informed participants that the purpose of the study was to demonstrate that these effects do not exist. These results suggest that demand characteristics can moderate_-but are not necessary for-facial feedback effects.

Study 1, nonetheless, had several potential limitations. The first limitation involves the fact that experimenters were not blind to participants' conditions. Consequently, the experimenters may have unknowingly exhibited experimenter bias (Rosenthal \& Fode, 1963). This experimenter bias could have been particularly problematic in the nil-hypothesis condition. If experimenters privately believed that facial feedback effects are real, they may have unknowingly signaled that they disagreed with the prediction communicated in the nilhypothesis condition. If so, observed facial feedback effects in that condition may have been driven entirely by participants who doubted that the experimenter truly hypothesized nil effects. Separately, experimenter bias could have also weakened the validity of the measure of participants' awareness of the communicated hypothesis by biasing how experimenters conducted the funnel debriefing and interpreted participants' responses.

The second limitation involves the fact that we led participants in the nil-hypothesis condition to believe that researchers disagreed about facial feedback effects. Specifically, participants in this condition were told that (a) some researchers believe that posing facial expressions influences emotion, but that (b) we believe this is not true (emphasis added). When faced with this disagreement, some participants may have opted to believe the other researchers' hypothesis (rather than our own competing prediction). This could have limited the effectiveness of the nil-hypothesis manipulation, causing us to erroneously observe evidence for facial feedback effects in this condition. We address these two limitations in Study 2. 


\section{Figure 2.}

Study 1 self-reported happiness and anger after posing happy, neutral, and angry facial expressions in the positive-hypothesis, no-hypothesis, and nil-hypothesis conditions.

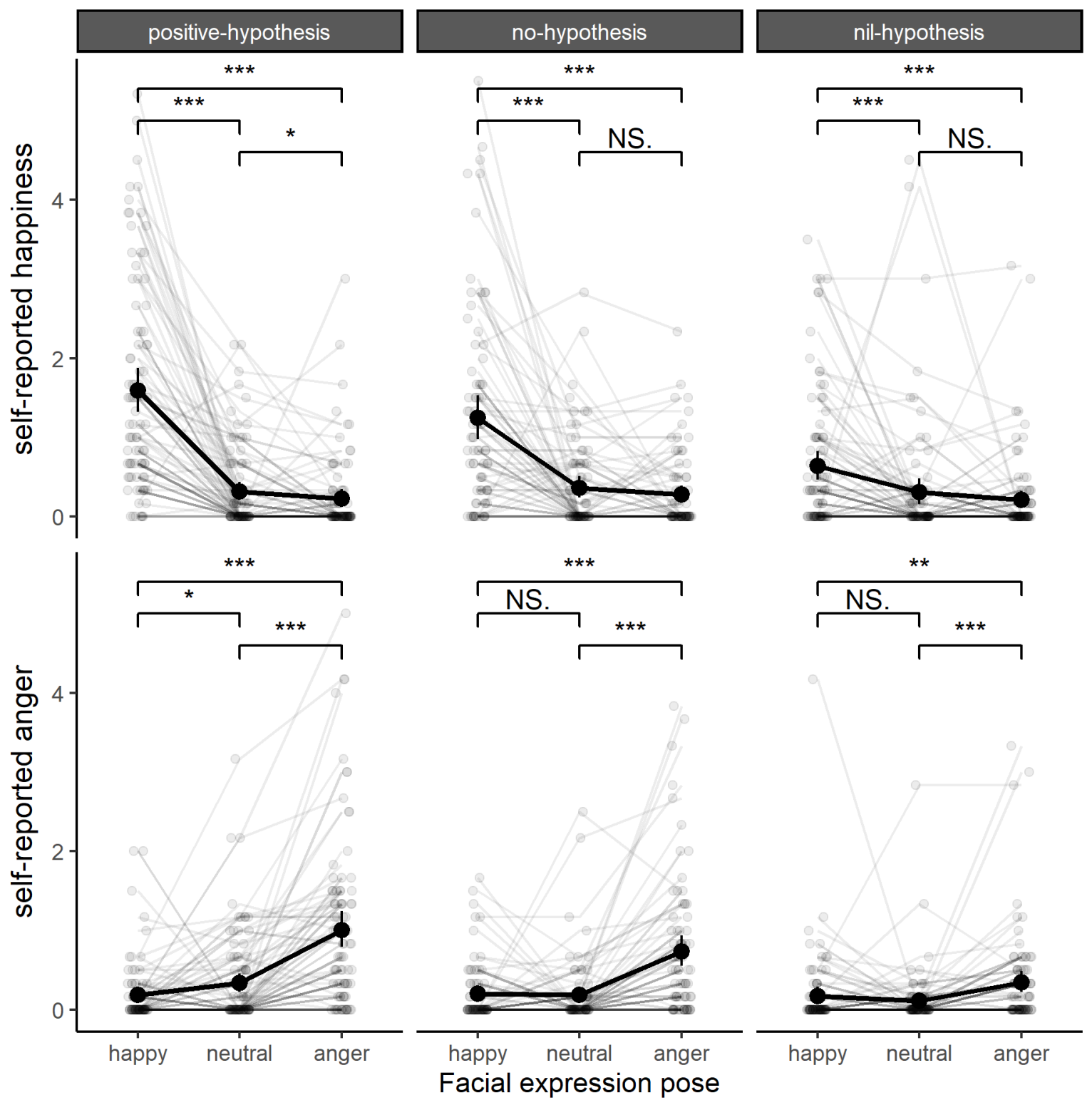

Note. Grey points represent jittered participant-level observations, bolded black circles represent group-level means, and bolded black vertical lines represent group-level standard errors.

Wilcoxon tests were used for all pairwise comparison tests.

$* p<.05 ; * * p<.01 ; * * * p<.001$ 


\section{Study 2}

We made two changes in Study 2. First, to prevent experimenter bias, we had participants complete the study online without interacting with an experimenter. Second, to improve the nilhypothesis condition, we removed information about other researchers' beliefs.

We once again predicted that facial feedback effects would be moderated by demand characteristics. However, based on the evidence of facial feedback effects in the Study 1 nilhypothesis condition, we updated our pre-registered predictions and hypothesized that we would again observe relatively small facial feedback effects in the nil-hypothesis condition.

\section{Method}

\section{Participants and design}

We used the same 3 (Pose: happy, angry, neutral) x 2 (Block: first or second) x 3 (Demand: positive-hypothesis, no-hypothesis, nil-hypothesis) mixed design from Study 1, with Demand manipulated between-subjects. Based on a power simulation with conservative effect size estimates from Study 1, we planned to recruit at least 180 participants to achieve (a) $\approx 99 \%$ power to detect the hypothesized Pose by Demand interaction, and (b) $>80 \%$ power to detect the hypothesized Pose effects within each level of Demand (see https://osf.io/uf68k/ for more details). One hundred and ninety-seven psychology students from the University of Tennessee completed the online experiment in exchange for partial course credit between July $29^{\text {th }}, 2020$, and September $8^{\text {th }}, 2020$. Per our pre-registered analysis plan, we excluded 5 participants who failed attention checks, leaving data from 192 participants (71\% women, 28\% men, 1\% other; $\left.M_{\text {age }}=18.60, S D_{\text {age }}=1.27\right)$. 


\section{Procedure}

Participants completed the online experiment via Qualtrics. Participants were told that they were participating in a study on physical movements and that they were randomly assigned to complete six face movements. (In actuality, all participants completed the same six facial movements.) Participants were then assigned to one of three Demand conditions using the random assignment feature in Qualtrics. Participants in the no-hypothesis condition $(n=65)$ began the facial expression poses without further instruction. Participants in the positivehypothesis $(n=67)$ and nil-hypothesis $(n=60)$ conditions received the following message (nilhypothesis message in brackets):

"We believe that emotions cause facial expressions - but that posing facial expressions can also [can not] cause emotions. For example, happiness makes you smile, but smiling can also [can not] make you happy. Likewise, anger makes you scowl, but scowling can also [can not] make you angry. The purpose of this study is to generate scientific evidence that facial expressions can [can not] cause emotions."

Like Study 1, participants completed two blocks of poses, each which included the same instructions to pose happy, angry, and neutral facial expressions in random order. After each pose, participants completed the same modified Discrete Emotions Questionnaire from Study 1 (overall happiness $a=.86$; overall anger $a=.88$ ), completed filler items about how difficult they found the posing task and how concentrated they were, and completed an attention check item that instructed participants to choose a specific response option (e.g., “please choose 'slightly' as your response to this question").

To check that participants were more aware we were testing facial feedback effects in the positive- and nil-hypothesis conditions (vs. the no-hypothesis condition), we asked participants 
to describe the purpose of the study after the two blocks of poses. Two independent coders who were blind to participants' conditions and the study results reviewed these responses and rated the degree to which each participant was aware we were testing facial feedback effects $(0=$ "not at all aware" to $4=$ "completely aware"). Coder ratings were similar (ICC $=.77,95 \%$ CI [.70, $.82])$, so we averaged them to form an awareness score.

To assess how well they completed the facial pose tasks, participants then reported the extent to which they followed instructions during the happy, angry, and neutral posing tasks $(0=$ "not at all" to $6=$ "an extreme amount"). To attenuate social desirability motives, we encouraged participants to respond truthfully and informed them they would not be penalized for indicating that they did not follow instructions. The University of Tennessee Institutional Review Board approved the experimental protocol.

\section{Results}

\section{Awareness that Experiment Tested Facial Feedback Effects}

Like Study 1, results from a one-way ANOVA confirmed that awareness ratings varied among the Demand conditions, $F(2,189)=6.81, p=.001$. Participants were less aware we were testing facial feedback effects in the no-hypothesis condition $(M=2.63, S D=1.69)$ than the positive-hypothesis $(M=3.33, S D=1.28), t(189)=-2.85, p=.005, M_{\text {diff }} 95 \%$ CI $[-1.18,-0.21]$, and nil-hypothesis conditions $(M=3.50, S D=1.19), t(189)=-3.45, p<.001, M_{\text {diff }} 95 \% \mathrm{CI}[-$ $1.37,-0.37]$. Awareness in the latter two conditions did not differ, $t(189)=-0.69, p=.49, M_{\text {diff }}$ 95\% CI [-0.67, 0.32]. 


\section{Adherence to Facial Posing Task Instructions}

It was not possible to covertly record participants during the facial posing tasks in this online study. Nonetheless, participants reported that they followed the instructions extremely closely for the happy $(M=5.26, S D=0.82)$, neutral $(M=5.41, S D=0.76)$, and angry $(M=5.29$, $S D=0.77)$ posing tasks.

\section{Self-Reported Emotion Experience}

We modeled emotion reports using the same linear mixed-effects regression approach used in Study 1. The primary effect of interest was the Pose by Demand interaction, but we report results from the full models in the Supplemental Materials. We additionally report modelestimated marginal means, pairwise comparison confidence intervals, and effect sizes in the Supplemental Materials.

Self-Reported Happiness. Replicating Study 1, there was a significant Pose by Demand interaction, $F(4,945)=3.18, p=.01$. As depicted in the top panel of Figure 3, the effect of Pose on self-reported happiness was largest in the positive-hypothesis condition, $F(2,945)=96.83, p$ $<.001$, second largest in the no-hypothesis condition, $F(2,945)=55.75, p<.001$, and smallest in the nil-hypothesis condition, $F(2,945)=36.35, p<.001$. In all Demand conditions, participants reported more happiness after posing happy vs. neutral and angry expressions (Figure 3).

Self-Reported Anger. Unexpectedly, we did not find significant evidence of a Pose by Demand interaction for anger reports, $F(4,945)=1.58, p=.18$. Nevertheless, the effect of Pose on self-reported anger appeared to be largest in the positive-hypothesis condition, $F(2,945)=$ $50.21, p<.001$, second largest in the no-hypothesis condition, $F(2,945)=43.74, p<.001$, and smallest in the nil-hypothesis condition $F(2,945)=19.88, p<.001$. In all three Demand 
conditions, participants reported more anger after posing angry vs. neutral and happy expressions (Figure 3). Taken together, these results do not provide clear evidence that the effect of posed expressions on anger is moderated by demand characteristics. More importantly, though, the observed effect of Pose in the nil-hypothesis condition suggests that the effects of facial feedback on anger were not entirely driven by demand characteristics.

\section{Discussion}

Study 2 largely replicated Study 1. Demand characteristics moderated the effects of posed expressions on happiness, but not anger. Most importantly, though, Study 2 consistently indicated that the effects of posed expressions on happiness and anger were significant in all levels of our demand characteristics manipulation. This includes the nil-hypothesis condition, where participants were told that we sought to demonstrate that facial poses do not impact emotions. Study 2 also suggests that these results are not driven by experimenter bias or ambiguity in the nil-hypothesis manipulation.

Unfortunately, our reliance on participants from the Southeastern United States in Studies 1-2 limits the generalizability of the findings (Henrich, Heine, \& Norenzayan, 2010). We begin to address this limitation in Study 3. 


\section{Figure 3.}

Study 2 self-reported happiness and anger after participants posed happy, neutral, and angry facial expressions in the positive-hypothesis, no-hypothesis, and nil-hypothesis conditions.

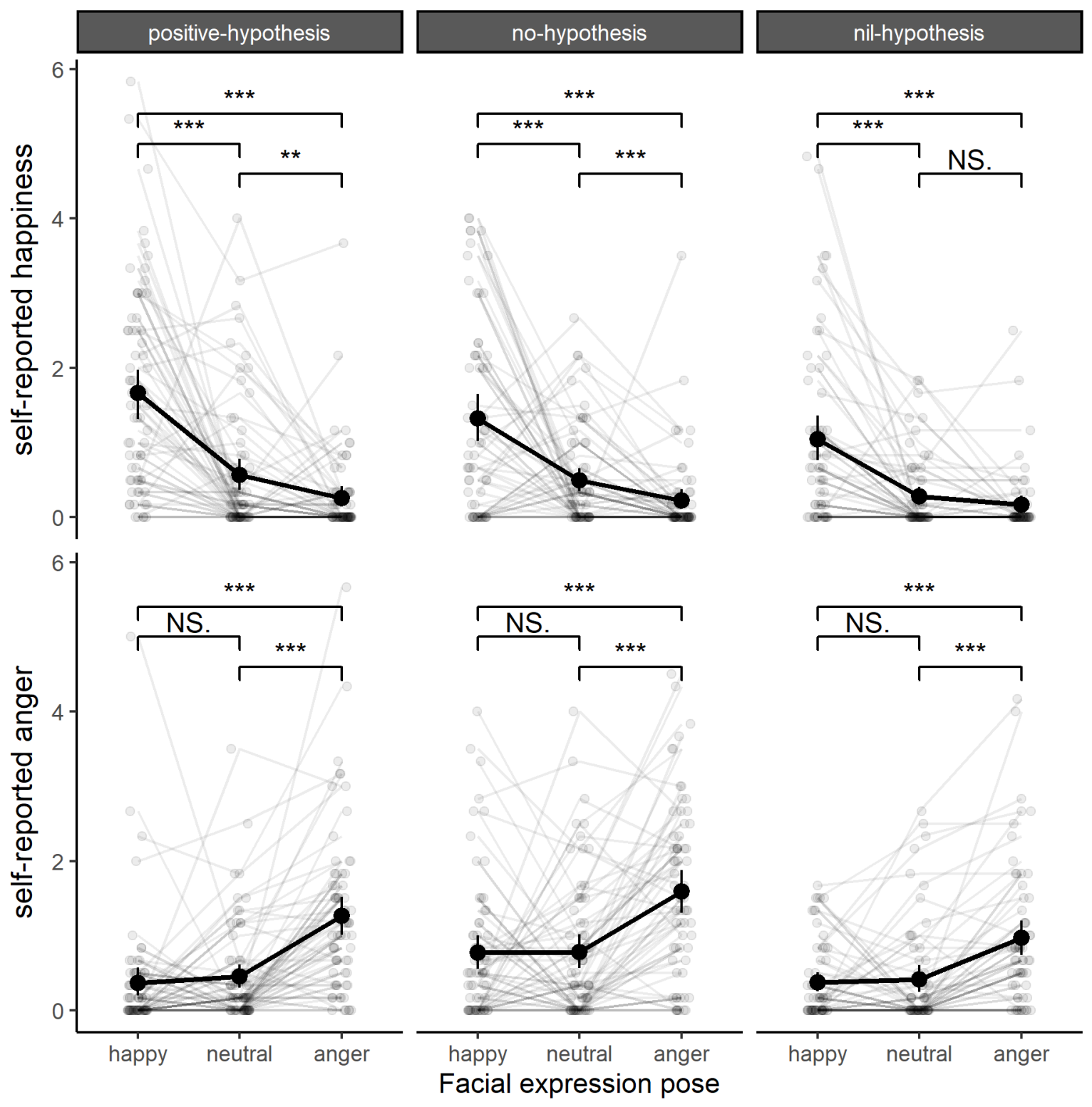

Note. Grey points represent jittered participant-level observations, bolded black circles represent group-level means, and bolded black vertical lines represent group-level standard errors.

Wilcoxon tests were used for all pairwise comparison tests.

$* p<.05 ; * * p<.01 ; * * * p<.001$ 


\section{Study 3}

\section{Method}

We conducted Study 3 with a university sample in Kenya. To maintain high statistical power with a relatively small participant pool, we did not include a no-hypothesis condition. We used a 3 (Pose: happy, angry, neutral) x 2 (Block: first or second) x 2 (Demand: positivehypothesis or nil-hypothesis) mixed design, with Demand manipulated between-subjects. Of course, removing the no-hypothesis condition precluded us from confirming that the demand characteristics manipulation increased awareness of our interest in testing facial feedback effects. Nevertheless, given that Studies 1 and 2 provided consistent evidence of the efficacy of the manipulation, we believe that this tradeoff was reasonable.

Based on a power simulation with conservative parameter estimates from Study 1, we planned to recruit at least 120 participants to achieve (a) $99 \%$ power to detect our hypothesized Pose by Demand characteristics interaction, and (b) $>80 \%$ power to detect our hypothesized Pose effects within each level of Demand (for more details, see https://osf.io/uf68k/). One hundred and fifty students from the United States International University-Africa completed the online experiment between August $8^{\text {th }}$ and $17^{\text {th }}, 2020$. We excluded 19 participants who failed attention checks, which left data from 131 participants $\left(n_{\text {positive-hypothesis }}=62, n_{\text {nil-hypothesis }}=69\right.$, $76 \%$ women, $22 \%$ men, $2 \%$ other, $\left.M_{\text {age }}=26.80, S D_{\text {age }}=8.07\right)$.

\section{Procedure}

Study 3 was conducted in English, which is one of two official languages in Kenya and the language of instruction in most Kenyan universities. All procedures were identical to Study 2 with two exceptions. First, as mentioned previously, we did not include a no-hypothesis condition. Second, we replaced the word "scowl" with the phrase "scowl or frown" throughout 
the experiment. We made this change because two classroom surveys ( $n$ 's $=21$ and 29) indicated that Kenyan students were less familiar with the term "scowl" and used it interchangeably with the term "frown" to describe the expression associated with anger.

\section{Results}

\section{Adherence to Facial Posing Task Instructions.}

The online protocol prevented us from recording participants during the facial posing tasks. Nevertheless, participants reported that they followed the instructions for the happy $(M=$ $5.03, S D=1.00)$, neutral $(M=5.02, S D=1.12)$, and angry $(M=5.07, S D=1.02)$ posing tasks extremely closely.

\section{Self-Reported Emotional Experience}

We modeled self-reported happiness (overall $a=.84$ ) and anger (overall $a=.86$ ) using the same linear mixed-effects regression approach used in Studies 1 and 2. The primary analysis of interest was the Pose by Demand interaction, but we report results from the full models in the Supplemental Materials. We additionally report model-estimated marginal means, pairwise comparison confidence intervals, and effect sizes in the Supplemental Materials.

Self-Reported Happiness. Replicating Studies 1 and 2, there was a significant Pose by Demand interaction, $F(2,645)=5.92, p=.003$. As depicted in the top panel of Figure 4 , the effect of Pose on self-reported happiness was larger in the positive-hypothesis, $F(2,645)=$ $62.00, p<.001$, than nil-hypothesis condition $F(2,645)=23.74, p<.001$. In both Demand conditions, participants reported more happiness after posing happy vs. neutral and angry expressions (Figure 4). As with the U.S. samples in Studies 1 and 2, these results with a Kenyan sample confirm that demand characteristics moderated, but did not fully account for, the effects of posed expressions on happiness. 
Self-Reported Anger. Replicating Study 1, there was a significant Pose by Demand interaction, $F(2,645)=10.46, p<.001$. As depicted in the bottom panel of Figure 3, the effect of Pose on self-reported anger was larger in the positive-hypothesis, $F(2,645)=85.22, p<.001$, than nil-hypothesis condition, $F(2,645)=26.45, p<.001$. Once again, in both Demand conditions, participants reported more anger after posing angry vs. neutral and happy expressions (Figure 4). These results once again suggest that the effects of posed expressions on anger are not entirely driven by demand characteristics. 


\section{Figure 4.}

Study 3 self-reported happiness and anger after participants posed happy, neutral, and angry facial expressions in the positive-hypothesis and nil-hypothesis conditions.

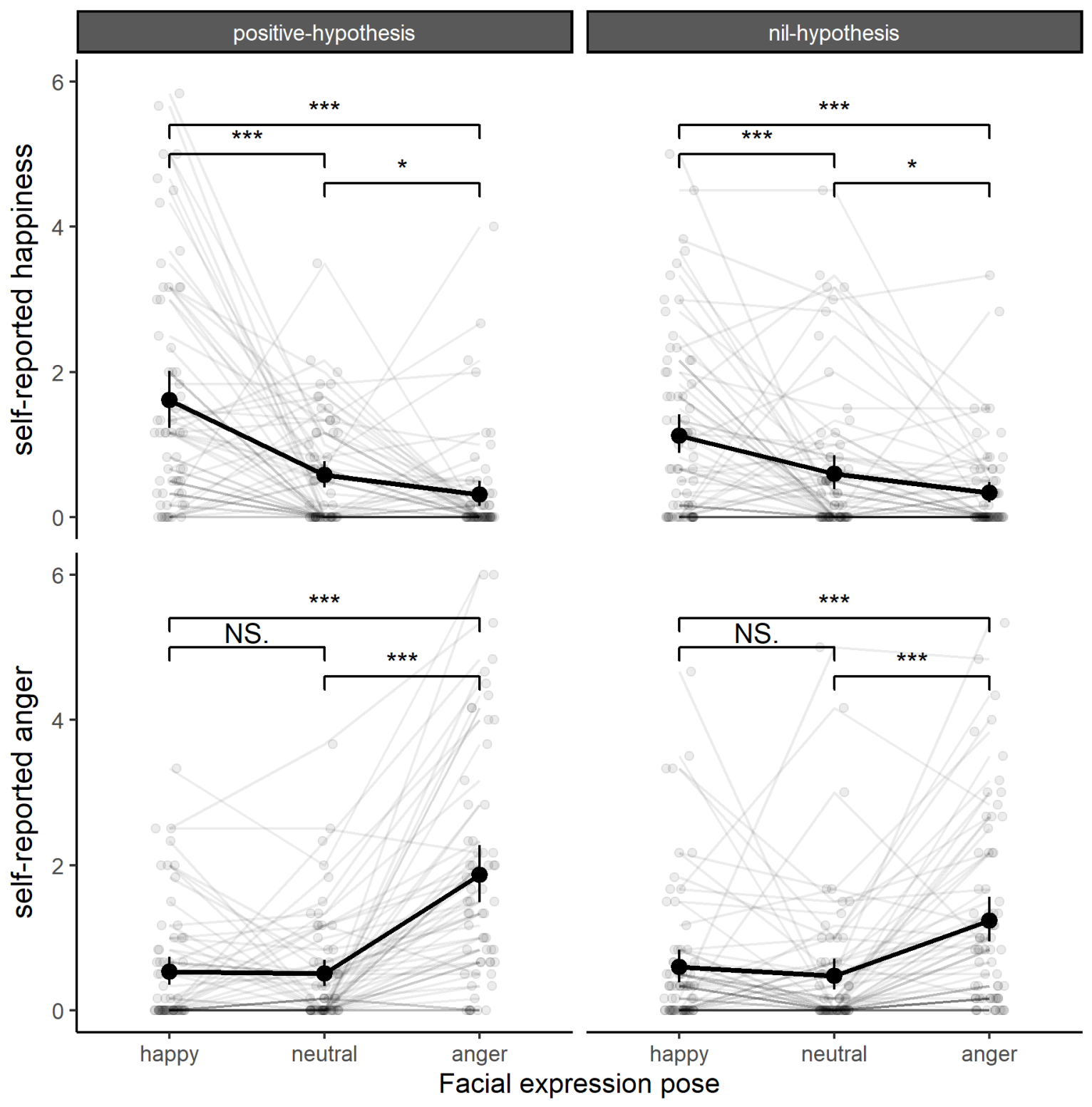

Note. Grey points represent jittered participant-level observations, bolded black circles represent group-level means, and bolded black vertical lines represent group-level standard errors.

Wilcoxon tests were used for all pairwise comparison tests.

${ }^{*} p<.05 ; * * p<.01 ; * * * p<.001$ 


\section{Overview of Studies 4-6}

Studies 1-3 provide evidence that demand effects do not completely account for facial feedback effects. In Studies 4-6, we next examined the role of participants' beliefs about facial feedback. We did so by capitalizing on ancillary measures of participants' beliefs that we collected in Studies 1-3. We conducted a pooled re-analysis of those data to investigate (a) whether demand characteristics impacted their beliefs in facial feedback effects, and (b) whether facial feedback effects were moderated by these beliefs. In Studies 5 and 6, we conducted additional tests by measuring participants' beliefs about facial feedback either before or after the study session.

\section{Study 4: A Re-Analysis of Pooled Data from Studies 1-3}

Experimenters in Study 1 rated the extent to which each participant believed that posing facial expressions could influence emotion based on funnel debriefings administered at the end of each session $(0=$ strong disbelief; $1=$ slight disbelief; $2=$ unsure; $3=$ slight belief; $4=$ strong belief). At the end of each session in Studies 2 and 3, participants self-reported their beliefs about facial feedback effects using the same scale.

We categorized participants based on whether they believed ( 4 or 3 on the aforementioned scale; $n=344 ; 63 \%$ of sample), were unsure if ( 2 on the aforementioned scale; $n$ $=48 ; 9 \%$ of sample), or did not believe ( 1 or 0 on the aforementioned scale; $n=154 ; 28 \%$ of sample) posing facial expressions can influence emotions. This approach of trichotomizing a continuous variable has some limitations (Altman \& Royston, 2006). However, inferences were similar regardless of whether we modeled belief as a trichotomous or continuous variable. (See Supplemental Materials for treatment of belief as a continuous variable.) Furthermore, as described below, Studies 5 and 6 used trichotomous measures of beliefs about facial feedback 
effects. Thus, to better compare results across studies, we focus here on the trichotomized belief variable.

\section{Results}

\section{The Effects of Demand Characteristics on Participants' Beliefs in Facial Feedback Effects}

To examine whether demand characteristics impacted participants' beliefs about facial feedback, we modeled the probability that participants were believers, unsure, or nonbelievers using multinomial regression with Demand as a predictor. As shown in Table 1, participants were more likely to be believers than nonbelievers in the positive hypothesis $(\beta=1.45, z=7.95$, $p<.001, O R=4.27)$ and no-hypothesis conditions $(\beta=1.02, z=4.78, p<.001, O R=2.77)$ and this pattern did not significantly differ between the two conditions, $\beta=0.43, z=1.55, p=$ $.12, O R=1.54$. However, relative to the no-hypothesis condition, this pattern was weakened in the nil-hypothesis condition $(\beta=-0.85, z=-3.29, p=.001, O R=0.43$ ), where participants were similarly likely to be believers than non-believers $(\beta=0.17, z=1.16, p=.25, O R=1.18)$. These results indicated that the nil-hypothesis condition decreased participants' beliefs in facial feedback relative to the other two conditions.

To examine the extent to which demand characteristics and participant beliefs were distinct, we examined how often participants' beliefs about facial feedback effects matched our communicated hypothesis. Results indicated that they often did not (Table 1). For instance, although $42 \%$ of participants in the nil-hypothesis condition did not believe in facial feedback effects, 50\% did. In other words, half the participants in the nil-hypothesis condition indicated that they personally believed facial feedback effects are real—despite being told that the experimenter hoped to demonstrate they were not. 
Taken together, these results suggest that demand effects and placebo effects are related but distinct (see Figure 1). This distinction raises the possibility that placebo effects may drive facial feedback effects, even in the absence of demand characteristics.

\section{Table 1.}

Frequency (and approximate percentage) of participants across Studies 1-3 in each Demand condition who believed, were unsure, or did not believe that facial feedback impacts emotion.

\begin{tabular}{llll}
\hline Demand condition & Believer & Unsure & Nonbeliever \\
\hline Positive-hypothesis & $158(75 \%)$ & $15(7 \%)$ & $37(18 \%)$ \\
No-hypothesis & $83(65 \%)$ & $15(12 \%)$ & $30(23 \%)$ \\
Nil-hypothesis & $103(50 \%)$ & $18(9 \%)$ & $87(42 \%)$ \\
\hline
\end{tabular}

\section{The Moderating Role of Participants' Beliefs about Facial Feedback Effects}

To test the moderating role of participants' beliefs about facial feedback, we modeled self-reported happiness and anger using linear mixed-effects regression with Pose, Block, Belief, and their higher-order interactions entered as effect-coded fixed-effect factors, random-intercepts for each participant, and Type III Sums of Squares. $F$-values are from ANOVA tables with Satterthwaite degrees of freedom. The primary effect of interest was the Pose by Belief interaction, but we report results from full models in the Supplemental Materials. We used Wilcoxon tests for all pairwise comparison tests, and we additionally report model-estimated marginal means, pairwise comparison confidence intervals, and effect sizes in the Supplemental Materials.

For both self-reported happiness and anger, there were significant Pose by Belief interactions: happiness, $F(4,2715)=48.95, p<.001$; anger, $F(4,2715)=26.80, p<.001$. As shown in Figure 5, Pose effects were largest amongst participants who believed in facial 
feedback effects (happiness $F(2,2715)=536.69, p<.001$; anger $F(2,2715)=334.73, p<.001$ ) and smallest among participants who were unsure (happiness $F(2,2715)=24.43, p<.001$; anger $F(2,2715)=8.69, p<.001)$ or did not believe in facial feedback effects (happiness $F(2,2715)=$ $15.61, p<.001$; anger $F(2,2715)=24.11, p<.001)$. Follow-up pairwise comparisons indicated that, regardless of their beliefs about facial feedback effects, participants (a) reported more happiness after posing happy vs. neutral and angry expressions, and (b) reported more anger after posing angry vs. neutral and happy expressions (Figure 5). Notably, this includes participants who indicated that they did not believe in facial feedback effects, which is quite remarkable given that they exhibited facial feedback effects mere minutes before reporting their beliefs.

One limitation of the above analyses is that participants' beliefs were not completely independent from the hypothesis communicated to them (i.e., demand characteristics). For example, many participants who did not believe in facial feedback effects were in a positivehypothesis condition wherein they were informed that the experimenter was trying to demonstrate the opposite. This raises the possibility that the facial feedback effects among nonbelievers were driven by the subset of participants exposed to the positive-hypothesis message. This seems unlikely, however, given that there were more than twice as many nonbelievers in the nil-hypothesis condition (which diminished facial feedback effects) than the positive-hypothesis condition (which amplified facial feedback effects). Nonetheless, even when we limited analyses to the no-hypothesis condition (where hypotheses were not explicitly communicated to participants), we found preliminary evidence that nonbelievers reported (a) 
more happiness after posing happy than neutral or angry expressions, and (b) more anger after posing angry than neutral expressions. ${ }^{5}$

\section{Discussion}

A pooled re-analysis of Studies 1-3 provides evidence that demand and placebo effects are related but distinct. The hypothesis communicated to participants had some effects on participants' beliefs about facial feedback — and both appeared to moderate facial feedback effects. Nonetheless, many participants exhibited beliefs that were inconsistent with the communicated hypothesis. In any event, results indicated that placebo effects cannot fully account for observed facial feedback effects_-as facial feedback effects were observed even among participants who did not believe in these effects.

Study 4, however, has two main limitations. First, we assessed beliefs at the end of the study sessions. Consequently, participants' beliefs about facial feedback effects may have been impacted by the extent to which they experienced genuine facial feedback effects during the study. Consequently, what appears to be beliefs influencing facial feedback effects could be facial feedback effects influencing beliefs. Second, we measured whether participants believed that facial feedback influences emotion in general. However, open-ended feedback revealed that participants' beliefs about facial feedback effects can vary by emotion. For example, some participants reported they believed that posing happy—but not angry—expressions can impact

${ }^{5}$ When examining the role of participants' belief about facial feedback in the no-hypothesis condition in Study 4, we were primarily interested in whether participants who were believers, unsure, or nonbelievers reported (a) higher levels of happiness after posing happy vs. neutral and happy vs. angry expressions, and (b) higher levels of anger after posing angry vs. neutral and angry vs. happy expressions. All but one of these contrasts were significant. The one exception was the comparison of anger reports when nonbelievers posed angry vs. happy expressions. Nonetheless, we believe that the preponderance of evidence suggests that participants' beliefs about facial feedback moderate- but do not fully account for-facial feedback effects. 
emotion. For such participants, our measures could not accurately capture their beliefs about emotion-specific facial feedback effects. We address these limitations in Studies 5 and 6. 


\section{Figure 5.}

Self-reported happiness and anger for Study 1-3 participants who posed happy, neutral, and angry expressions and either believed, were unsure, or did not believe in facial feedback effects.

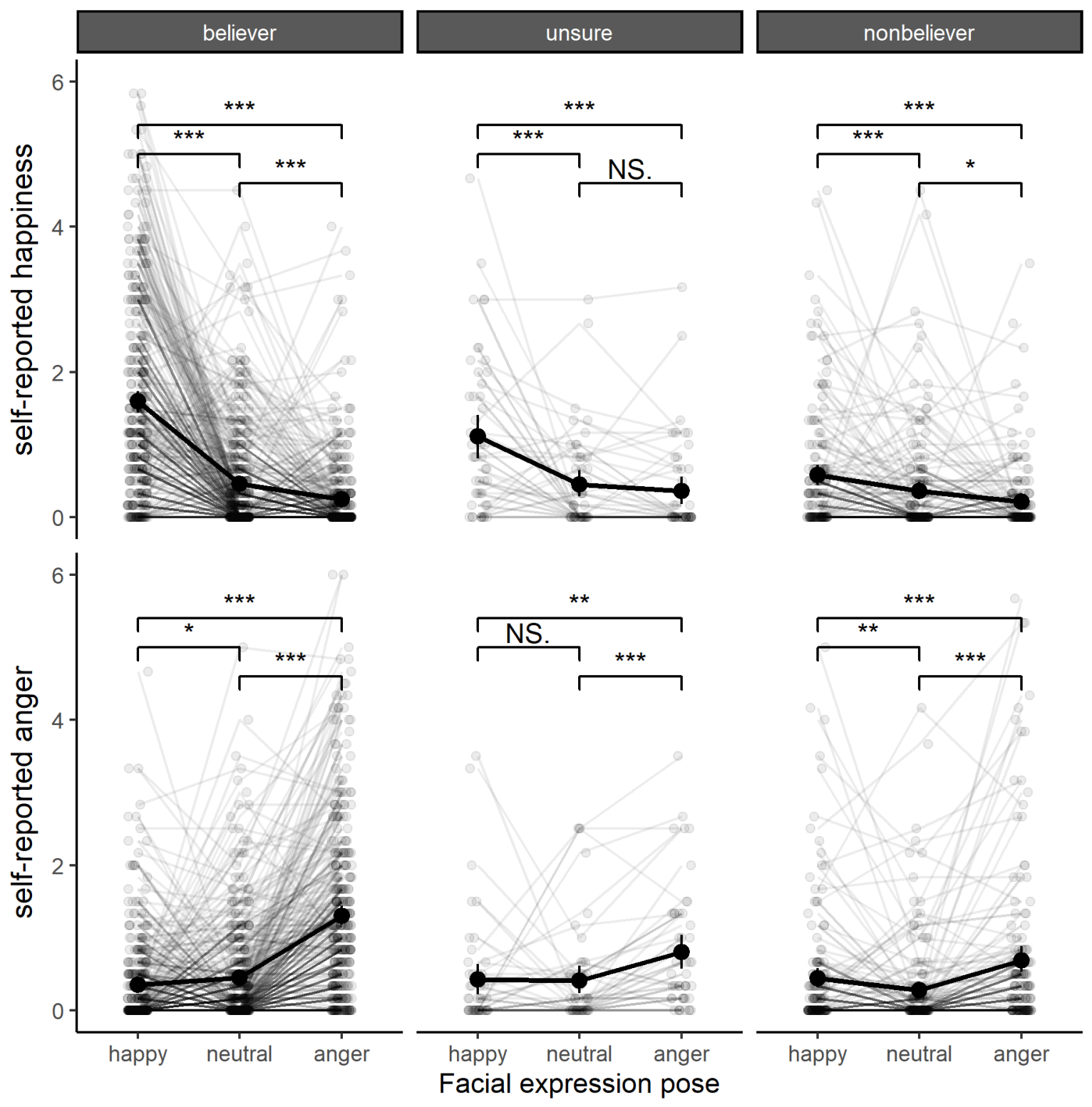

Note. Grey points represent jittered participant-level observations, bolded black circles represent group-level means, and bolded black vertical lines represent group-level standard errors.

Wilcoxon tests were used for all pairwise comparison tests.

$* p<.05 ; * * p<.01 ; * * * p<.001$ 


\section{Study 5}

Study 5 was a pre-registered examination of the extent to which facial feedback effects are moderated by participants' beliefs about facial feedback. To isolate the moderating role of participants' beliefs, we did not communicate information about our hypothesis. Furthermore, we measured participants' beliefs prior to the study sessions. To improve the measurement of participants' beliefs, we developed an assessment that (a) distinguished between the effects of facial feedback on happiness and anger, and (b) included multiple items about those beliefs.

\section{Pre-study survey of beliefs about facial feedback}

We conducted Study 5 with Introduction to Psychology students from the University of Tennessee. Students were asked to complete a pre-screen survey that included our assessment of facial feedback beliefs (amid questionnaires related to other studies in which they might later participate). Many students completed the survey at the beginning of the semester, though some completed it later in the semester and others did not complete it at all.

We asked students four questions regarding their beliefs about facial feedback. Two items targeted beliefs about the effects of posed happy expressions on happiness: (1) "Imagine that you were in a relatively neutral emotional state and then you posed a happy facial expression. Do you think you would feel more happy after posing the happy facial expression?" (Yes / No / I'm not sure); and (2) "Do you personally think that people can make themselves feel happy by posing a happy facial expression?” (Yes / No / I'm not sure). The assessment also included two similarly worded questions regarding the effects of posed angry expressions on anger.

A total of 620 students completed the pre-study survey (a subset of whom later completed our main experiment). For the measures of both happy and angry facial feedback beliefs, the 
majority of the 620 participants answered the two items consistently (e.g., answering "Yes" or "No" to both the first and second question). Specifically, $63 \%(\kappa=.43)$ of participants answered the two happy facial feedback belief questions consistently, and $61 \%(\kappa=.42)$ of participants answered the two angry facial feedback belief questions consistently. For happy and angry facial feedback beliefs, we categorized participants as (a) believers if they answered "Yes" to both associated questions, (b) non-believers if they answered "No" to both associated questions, and (c) "Unsure" in all other scenarios. For happy facial feedback effects, 210 (34\%) participants were believers, 296 (48\%) were unsure, and 114 (18\%) were nonbelievers. For angry facial feedback effects, 152 (25\%) participants were believers, 324 (52\%) were unsure, and 114 (23\%) were nonbelievers.

\section{Method}

\section{Participants and design}

The main experiment used a 3 (Pose: happy, angry, neutral) x 2 (Block: first or second) $\mathrm{x}$ 3 (Belief: believer, unsure, nonbeliever) mixed design. Belief was measured during the pre-study survey and varied between-subjects. Based on a power simulation with conservative parameter estimates from Study 4, we aimed to recruit 60 facial feedback believers and 60 facial feedback nonbelievers to achieve (a) $>90 \%$ power to detect our hypothesized Pose by Belief interactions and (b) $>80 \%$ power to detect our hypothesized Pose effects for both believers and nonbelievers (for more details, see https://osf.io/uf68k/). However, because the participant pool closed at the end of the academic semester, we pre-registered that we would end data collection at the end of the Spring 2021 semester. We eventually used this latter stopping rule (but see Study 6 for a higher-power conceptual replication). 
One hundred and thirty-seven students completed the experiment in exchange for partial course credit between January 20 and May 5, 2021. We excluded participants who did not complete the pre-study belief measure $(n=12)$, completed the pre-study belief measure after completing the main experiment $(n=10)$, did not finish the main experiment $(n=8)$, or failed an attention check $(n=29)$. This yielded data from 78 participants $(59 \%$ women, $37 \%$ men, $4 \%$ other, $\left.M_{\mathrm{age}}=19.48, S D_{\mathrm{age}}=1.92\right)$. For happy facial feedback effects, $34(43.59 \%)$ participants were believers, $22(28.21 \%)$ were unsure, and 22 (28.21\%) were nonbelievers. For angry facial feedback effects, $20(25.64 \%)$ participants were believers, $33(42.31 \%)$ were unsure, and 25 $(32.05 \%)$ were nonbelievers. We originally planned to analyze data from only believers and nonbelievers. However, to facilitate comparisons to Study 4, we report data from unsure participants below. (Similar results were obtained when these participants were excluded.)

The duration between when participants completed the pre-study survey and main experiment varied $(\min =0$ days $; M d n=6$ days $; \max =99$ days $)$. Although we were concerned that participants' beliefs about facial feedback might have changed during the elapsed time, exploratory analyses did not suggest that this was the case. A multinomial logistic regression of belief on duration of delay did not indicate that participants' beliefs about happy, $\chi^{2}(2)=0.24, p$ $=.89$, or angry, $\chi^{2}(2)=2.08, p=.35$, facial feedback effects differed over the course of the semester. 


\section{Procedure}

Participants completed the online experiment via Qualtrics. To minimize the role of demand characteristics, we emphasized our uncertainty and agnosticism when introducing participants to the research question:

"In this study, we are examining whether or not posing facial expressions can impact emotion. We know that emotions can cause facial expressions - but we do not know if posing facial expressions can cause emotions. For example, we know that happiness makes you smile, but we do not know if posing a smile can make you happy. Likewise, we know that anger makes you scowl, but we do not know if posing a scowl can make you angry. This study is designed to test whether or not posing facial expressions can impact emotion."

Like Studies 1-3, participants completed two blocks of poses, each which included the same instructions to pose happy, angry, and neutral facial expressions in random order. After each pose, participants completed (a) the modified Discrete Emotions Questionnaire featured in Studies 1-3 (overall happiness $a=.81$; overall anger $a=.91$ ), (b) filler items about how difficult they found the posing task and how much they concentrated, and (c) an attention check item that instructed participants to choose a specific response option (e.g., "please choose 'slightly' as your response to this question").

After the two blocks of poses, we asked participants to rate the extent to which they followed instructions during the happy, angry, and neutral posing tasks $(0=$ "not at all" to $6=$ “an extreme amount"). To attenuate social desirability, we encouraged participants to respond truthfully by assuring them that they would not be penalized for indicating that they did not 
follow instructions. The University of Tennessee Institutional Review Board approved the experimental protocol.

\section{Results}

\section{Adherence to Facial Posing Task Instructions}

As in Studies 2 and 3, participants reported that they followed the instructions for the happy $(M=5.27, S D=0.89)$, neutral $(M=5.64, S D=0.56)$, and angry $(M=5.27, S D=0.94)$ posing tasks very closely.

\section{Self-Reported Emotional Experience}

As in Study 4, we modeled self-reported happiness and anger using linear mixed-effects regression. Models were fit with Pose, Block, and Belief entered as effects-coded, fixed-effect factors, all higher-order interactions, random-intercepts for participants, and Type III Sums of Squares. For self-reported happiness and anger, the corresponding emotion-specific facial feedback belief was used as the Belief factor. Specifically, for self-reported happiness, Belief was operationalized as belief in the effect of posed happy expressions on feelings of happiness. For self-reported anger, Belief was operationalized as belief in the effect of posed angry expressions on feelings of anger. The primary effects of interest were the Pose by Belief interactions, but we report results from the full models in the Supplemental Materials along with model-estimated marginal means, pairwise comparison confidence intervals, and effect sizes.

Self-Reported Happiness. As in Study 4, there was a significant Pose by Belief interaction, $F(4,375)=4.74, p<.001$. As depicted in the top panel of Figure 6 , the effect of Pose on self-reported happiness was largest among participants who believed, $F(2,375)=73.70$, $p<.001$, second largest among participants who were unsure, $F(2,375)=22.97, p<.001$, and smallest among participants who did not believe that posed expressions of happiness could 
impact feelings of happiness, $F(2,375)=12.14, p<.001$. Replicating Study 4, participants who were believers, unsure, and nonbelievers all reported more happiness after posing happy vs. neutral and angry expressions. These results confirm that participants' beliefs moderate, but do not fully account for, the effects of posed expressions on happiness.

Self-Reported Anger. There was a significant Pose by Belief interaction, $F(4,375)=$ 2.73, $p=.03$. Surprisingly, however, results did not indicate that the effects of Pose on selfreported anger were larger amongst participants who believed, $F(2,375)=21.69, p<.001$, vs. did not believe in angry facial feedback effects, $F(2,375)=23.09, p<.001$. Instead, the Pose by Belief interaction was driven by an unexpected decrease in the size of the Pose effect among participants who were unsure if posed expressions of anger could impact feelings of anger, $F(2$, $375)=8.73, p<.001$. Nevertheless, like Study 4, participants who were believers, unsure, and nonbelievers all reported more anger after posing angry vs. neutral and happy expressions. These results confirm that participants' beliefs about facial feedback effects do not fully account for the effects of posed expressions on anger. 


\section{Figure 6.}

Self-reported happiness and anger for Study 5 participants who posed happy, neutral, and angry expressions and either believed, were unsure, or did not believe in facial feedback effects.

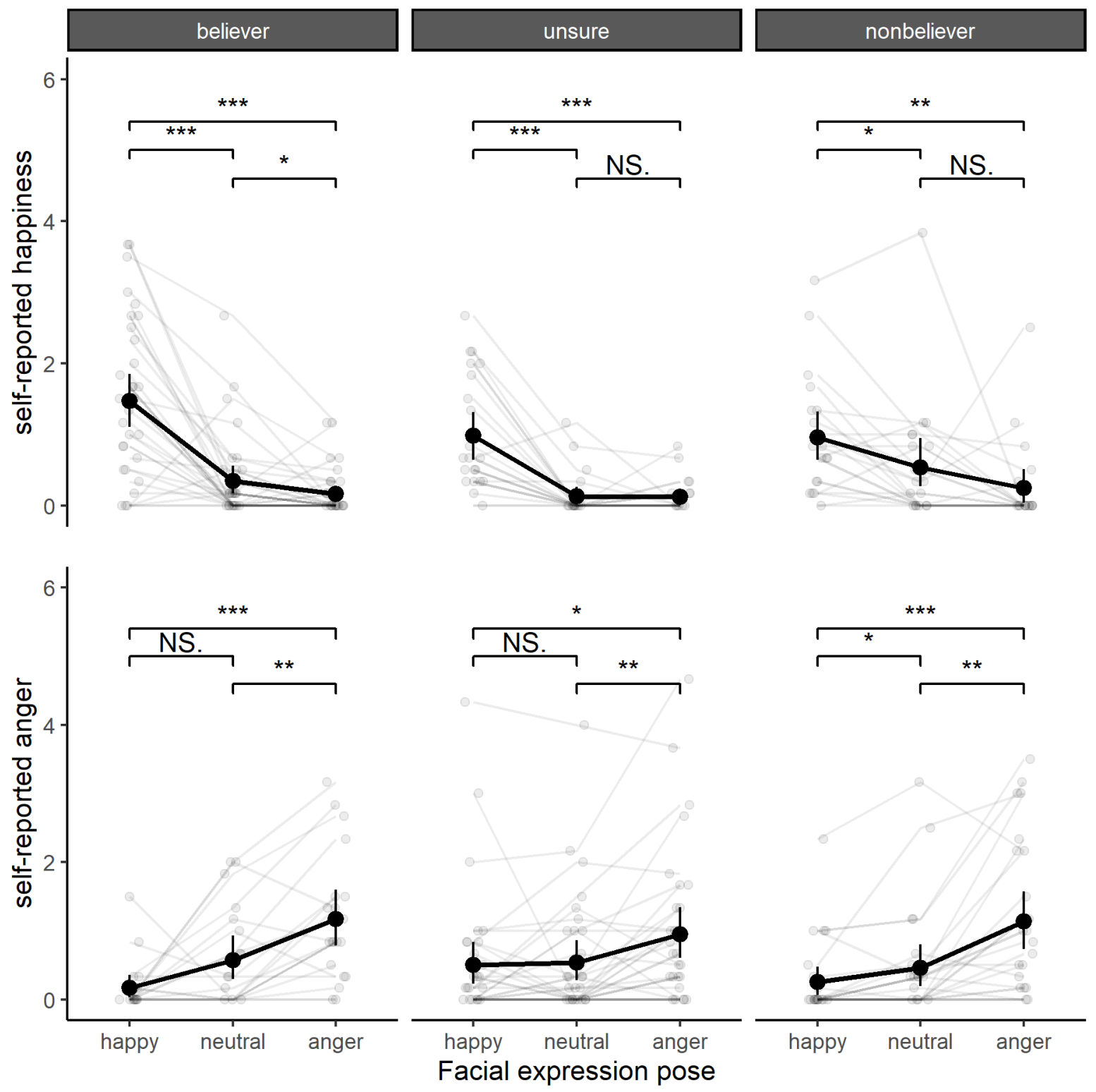

Note. Grey points represent jittered participant-level observations, bolded black circles represent group-level means, and bolded black vertical lines represent group-level standard errors.

Wilcoxon tests were used for all pairwise comparison tests.

$* p<.05 ; * * p<.01 ; * * * p<.001$ 


\section{Discussion}

Study 5 partially replicates Study 4. Like Study 4, the effect of posed facial expressions on self-reported happiness was larger among participants who believed in these effects (vs. were unsure about or did not believe). Unlike Study 4, however, we did not find the same pattern of results for self-reported anger. We did not find that the effect of facial feedback on self-reported anger differed among participants who believed vs. did not believe in these effects. Instead, we found that the effect of facial feedback on anger was smallest among participants who were unsure about these effects. Most importantly, however, facial feedback influenced both selfreported happiness and anger even among participants who did not believe in these effects. These results suggest that placebo effects do not fully account for facial feedback effects.

Study 5 has one notable limitation. To limit demand characteristics, we told participants we do not know if facial expressions can impact emotion. Unfortunately, stating our own uncertainty may have caused participants to doubt their own beliefs about facial feedback effects. This is most troubling for our inferences about facial feedback effects among participants who initially reported that they did not believe in these effects. Although we characterized these participants as "nonbelievers", it is possible that they were actually unsure about their beliefs after learning that we were uncertain about facial feedback effects. We address this limitation in Study 6, wherein we also collect a multi-country sample to facilitate cross-cultural generalizability. 


\section{Study 6}

Study 6 was a multi-country investigation of the extent to which facial feedback effects are moderated by participants' beliefs about these effects. Whereas we measured participants' beliefs about facial feedback before the experiment in Study 5 and after the experiment in Studies 1-3, we manipulated whether we measured participants' beliefs immediately before vs after the experiment in Study 6. We manipulated the time frame because each option has unique benefits and limitations. On one hand, measuring facial feedback beliefs at the beginning of the experiment may make these beliefs salient. On the other hand, measuring beliefs at the end of the experiment raises the possibility that beliefs changed because of experiences in the study. Manipulating the timeframe of measurement allowed us to formally examine whether associations between facial feedback effects and beliefs depended on when the beliefs were measured.

We made three additional methodological changes from Study 5. First, we did not inform participants of our uncertainty about facial feedback effects to address concerns that doing so in Study 5 may have altered participants' beliefs. Second, based on feedback from an anonymous reviewer, we manipulated the wording of the emotion measure. In the previous studies, we asked participants to report how they had felt during the preceding facial pose (i.e., a retrospective emotion report). A reviewer suggested that our results would be more compelling if we had asked participants to report how they were currently feeling (i.e., a current emotion report). We thus manipulated whether participants provided retrospective vs. current ratings of emotions. Third, to improve the generalizability of our results, we recruited English speakers from around the world to participate in our study. 


\section{Method}

\section{Participants and design}

Study 6 used a 3 (Pose: happy, angry, neutral) x 2 (Block: first or second) x 3 (Belief: believer, unsure, nonbeliever) x 2 (Belief Timing: before or after the experiment) x 2 (Emotion Report: retrospective or current) mixed design, with Belief, Belief Timing, and Emotion Report varied between-subjects. We acknowledge that this is a seemingly complex design. However, we pre-registered that we did not expect any effects involving the latter two factors. We did not expect effects involving Belief Timing because Studies 4 and 5 yielded similar results despite measuring beliefs at different times. Furthermore, we did not expect effects involving the type of emotion report because a meta-analysis by Coles et al. (2019) did not indicate that facial feedback effects were sensitive to the timing of the emotion measure.

Based primarily on the availability of funds, we planned to recruit 400 participants. The Study 5 power analysis indicated that this sample size provided high power to detect the hypothesized (a) Pose by Belief interactions and (b) Pose effects among people who believed, were unsure, or did not believe in facial feedback effects. Three hundred and ninety-seven Prolific workers completed the study in exchange for $\$ 2.75$ (\$11/hr) on July 7, 2021. Prolific workers were eligible to participate if they (a) spoke English, (b) had not previously completed a Prolific facial feedback experiment conducted by our team, (c) had completed between 10 and 10,000 studies on Prolific, and (d) had at least $90 \%$ of their Prolific study submissions accepted. These latter two inclusion criteria were used to ensure that the quality of participants' responses had been vetted by other researchers.

After excluding participants who failed an attention check $(n=31)$ or indicated that they did not complete the task correctly $(n=22)$ we were left with data from 344 participants $(37 \%$ 
women, $63 \%$ men, $<1 \%$ other, $\left.M_{\text {age }}=25.35, S D_{\text {age }}=6.90\right)$. These participants were located in the following countries: Portugal $(n=93)$, Poland $(n=47)$, Italy $(n=30)$, Mexico $(n=28)$, South Africa $(n=28)$, United Kingdom $(n=28)$, Spain $(n=16)$, Greece $(n=15)$, Chile $(n=11)$, Hungary ( $n=10)$, Czech Republic $(n=4)$, Estonia $(n=4)$, Ireland $(n=4)$, France $(n=3)$, Netherlands $(n=3)$, Australia $(n=2)$, Finland $(n=2)$, Israel $(n=2)$, Norway $(n=2)$, Slovenia $(n$ $=2)$, United States $(n=2)$, Belgium $(n=1)$, Canada $(n=1)$, Germany $(n=1)$, Latvia $(n=1)$,

New Zealand $(n=1)$, Sweden $(n=1)$, and Switzerland $(n=1)$. One participant did not report their location. For happy facial feedback effects, 117 (34\%) participants were believers, 157 $(46 \%)$ were unsure, and $70(20 \%)$ were nonbelievers. For angry facial feedback effects, 72 (21\%) participants were believers, 146 (42\%) were unsure, and 126 (37\%) were nonbelievers.

\section{Procedure}

Participants completed the online experiment via Qualtrics. To avoid altering their beliefs about facial feedback, we did not provide participants with explicit information about the true purpose of the study. Like Studies 1-5, participants completed two blocks of poses, each of which included the same instructions to pose happy, angry, and neutral facial expressions in random order. After each pose, participants completed a similar Discrete Emotions Questionnaire as in Studies 1-5. Depending on random assignment, participants either provided retrospective ratings (“As you were completing this trial's facial movement task, did you experience any of the following emotions?") or current ratings ("In this moment, are you currently experiencing any of the following emotions?") of the experienced happiness, anger, and fear $(0=$ "No, not at all" to 6 "Yes, at maximal strength"). Both the measures of retrospective (overall happiness $a=.87$; overall anger $a=.80$ ) and current (overall happiness $a=$ .93 ; overall anger $a=.75$ ) emotional experience exhibited good internal reliability. 
After reporting their emotions, participants completed filler items about how difficult they found the posing task, completed an attention check item that instructed them to choose a specific response option (e.g., "please choose 'slightly' as your response to this question”), and reported whether they completed the facial movement task correctly. After the two blocks of poses, participants reported the extent to which they followed instructions during the happy, angry, and neutral posing tasks $(0=$ "not at all" to $6=$ "an extreme amount").

We measured participants' beliefs about facial feedback effects using the same measure from Study 5. Depending on random assignment, participants completed the belief measure immediately before or after the experiment. The Stanford University Institutional Review Board approved the experimental protocol.

\section{Results}

\section{Adherence to Facial Posing Task Instructions}

As in Studies 2, 3, and 5, participants reported that they followed the instructions for the happy $(M=5.27, S D=0.70)$, neutral $(M=5.53, S D=0.70)$, and angry $(M=5.25, S D=0.77)$ posing tasks very closely.

\section{Beliefs About Facial Feedback}

Although beyond the original scope of our analysis plan, we used multinomial regression to examine whether the distribution of believers, unsure, and nonbelievers varied depending on whether we measured beliefs before or after the experiment (see Table 2). For beliefs about happy facial feedback effects, the patterns of belief varied depending on when these beliefs were measured, $\chi^{2}(2)=7.50, p=.02$. Participants were more likely to be unsure vs. believers in happy facial feedback effects when these beliefs were measured at the beginning $(\beta=0.54, z=3.10, p$ $=.002, O R=1.73)$ - but not the end $(\beta=0.04, z=0.26, p=.80, O R=1.05) —$ of the study, $\beta_{\text {time }}$ 
$=0.50, z=2.04, p=.04, O R=1.65$. Participants were more likely to be unsure vs. nonbelievers in happy facial feedback effects both when beliefs were measured at the beginning $(\beta=1.18, z=$ 5.37, $p<.001, O R=3.26)$ and end of the study $(\beta=0.47, z=2.43, p=.01, O R=1.60)$-but this was more pronounced when beliefs were measured at the beginning of the study, $\beta_{\text {time }}=0.71, z=$ $2.41, p=.02, O R=2.03$. This pattern of results suggests that participants were less likely to be unsure about happy facial feedback effects when beliefs were measured at the end of the study.

For beliefs about anger facial feedback effects, the patterns of belief did not significantly differ depending on when we measured beliefs, $\chi^{2}(2)=2.82, p=.24$. On average, participants were less likely to believe vs. be unsure $(\beta=-0.71, z=-4.91, p<.001, \mathrm{OR}=0.49)$ or not believe $(\beta=-0.56, z=-3.79, p<.001, O R=0.57)$ in anger facial feedback effects. Taken together, these results suggest that the timing of the belief measure had a significant effect on beliefs about happy—but not anger—facial feedback effects.

\section{Table 2.}

Frequency (and percentage) of Study 6 participants who reported either before or after the experiment that they believed, were unsure if, or did not believe that facial feedback impacted feelings of happiness and anger.

\begin{tabular}{lllll}
\hline Facial feedback belief & Belief measure timing & Believer & Unsure & Nonbeliever \\
\hline Happy facial feedback & Before experiment & $51(31 \%)$ & $88(53 \%)$ & $27(16 \%)$ \\
& After experiment & $66(37 \%)$ & $69(39 \%)$ & $43(24 \%)$ \\
Angry facial feedback & Before experiment & $34(20 \%)$ & $64(39 \%)$ & $68(41 \%)$ \\
& After experiment & $38(21 \%)$ & $82(46 \%)$ & $58(33 \%)$
\end{tabular}




\section{Self-Reported Emotional Experience}

Per our pre-registration plan, we modeled self-reported happiness and anger reports using linear mixed-effects regression with Pose, Block, Belief, and their higher-order interactions entered as effect-coded fixed-effect factors, random-intercepts for each participant, and Type III Sums of Squares. ${ }^{6}$ We focus primarily on the Pose by Belief interaction, but we report results from the full models in the Supplemental Materials along with model-estimated marginal means, pairwise comparison confidence intervals, and effect sizes.

Self-Reported Happiness. Replicating Studies 4-5, there was a significant Pose by Belief interaction, $F(4,1705)=20.47, p<.001$. As depicted in the top panel of Figure 7 , the effect of Pose on self-reported happiness was largest amongst participants who believed, $F(2$, $1705)=193.15, p<.001$, second largest among participants who were unsure, $F(2,1705)=$ $144.36, p<.001$, and smallest amongst participants who did not believe in happy facial feedback effects, $F(2,1705)=9.08, p<.001$. Importantly, participants of each belief type reported more happiness after posing happy vs. neutral and angry expressions. These results demonstrate once again that participants' beliefs moderate, but do not fully account for, the effects of posed expressions on happiness.

We did not find that these patterns differed depending on whether we measured beliefs before vs. after the experiment. Specifically, we did not observe a two-way interaction between Pose and Belief Timing, $F(2,1690)=0.93, p=0.40$, or a three-way interaction between Pose, Belief, and Belief Timing, $\mathrm{F}(4,1690)=0.81, p=0.52$. Likewise, we did not find that these

${ }^{6}$ We attempted to model random intercepts and slopes for country. Models containing random slopes did not converge because (a) some countries had a small number of observations, and (b) there was little-to-no between-country variability in many of the estimated effects. Models containing random intercepts for country yielded the same results as the simpler models described in the main text. 
patterns differed depending on whether participants reported retrospective or current emotions. Specifically, we did not observe a two-way interaction between Pose and Emotion Report, $F(2$, $1690)=1.42, p=.24$, or a three-way interaction between Pose, Belief, and Emotion Report, $F(4$, $1690)=0.93, p=.44$. Consequently, we have no reason to expect that our results hinge on such methodological details.

Self-Reported Anger. There was a significant Pose by Belief interaction, $F(4,1705)=$ 10.04, $p<.001$. As depicted in the top panel of Figure 7, the effect of Pose on self-reported anger was similarly large among participants who believed, $F(2,1705)=109.24, p<.001$, or were unsure, $F(2,1705)=109.31, p<.001$ about angry facial feedback effects. The effect of Pose was smallest among participants who did not believe in angry facial feedback effects, $F(2,1705)=$ $50.11, p<.001$. Nonetheless, participants of each belief type reported more anger after posing angry vs. neutral and happy expressions. These results demonstrate, once again, that participants' beliefs can moderate, but do not fully account for, the effects of posed expressions on happiness.

As with happiness reports, we did not find that these patterns of results differed depending on whether we (a) measured beliefs before vs. after the experiment or (b) whether participants reported retrospective or current emotions. We did not observe a two-way interaction between Pose and Belief Timing, $F(2,1690)=1.48, p=0.23$, a three-way interaction between Pose, Belief, and Belief Timing, $F(4,1690)=0.39, p=0.81$, a two-way interaction between Pose and Emotion Report, $F(2,1690)=2.56, p=0.08$, or a three-way interaction between Pose, Belief, and Emotion Report, $F(4,1690)=1.20, p=0.31$. 


\section{Figure 7.}

Self-reported happiness and anger for Study 6 participants who posed happy, neutral, and angry expressions and either believed, were unsure, or did not believe in facial feedback effects.

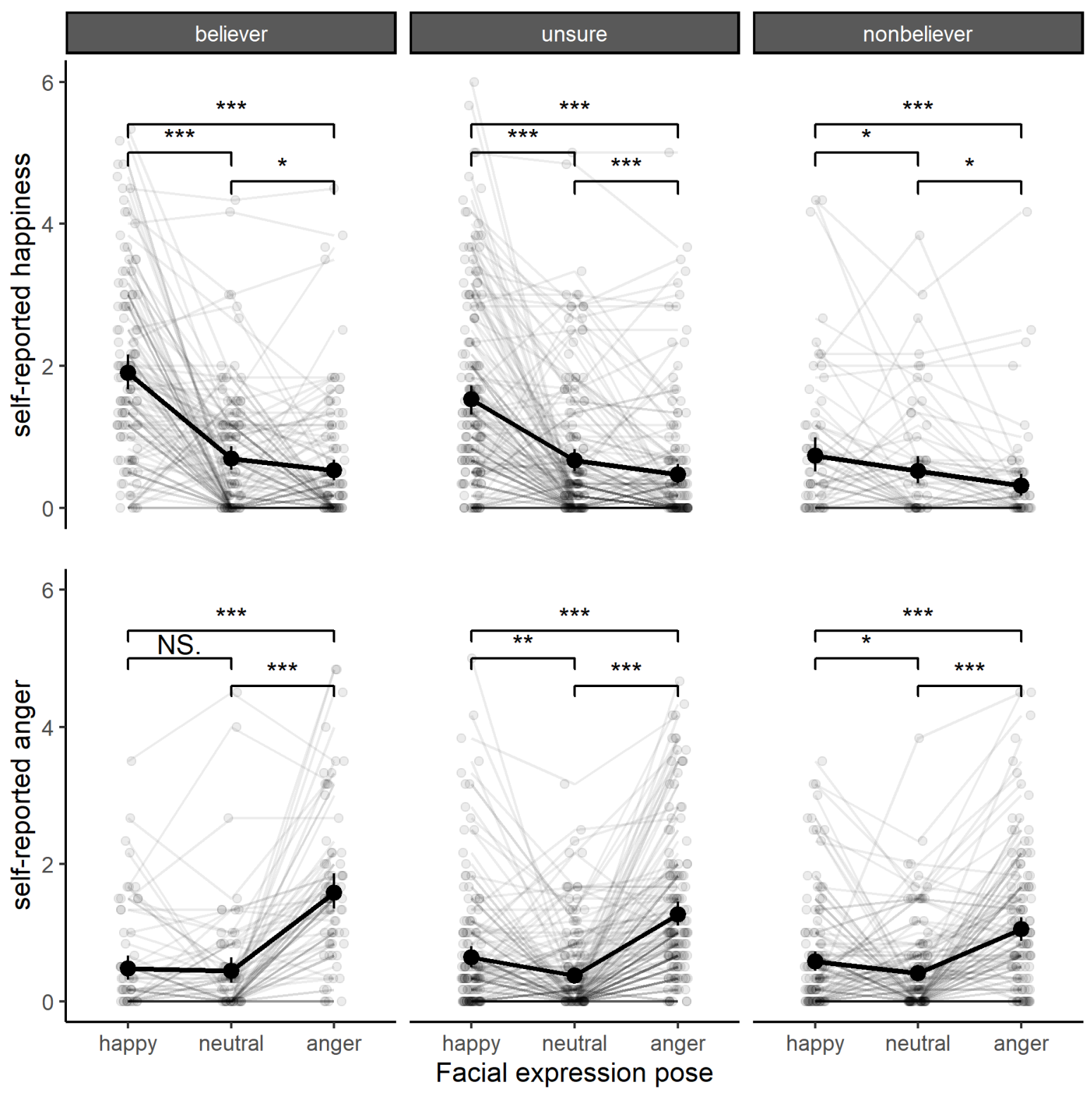

Note. Grey points represent jittered participant-level observations, bolded black circles represent group-level means, and bolded black vertical lines represent group-level standard errors.

Wilcoxon tests were used for all pairwise comparison tests.

${ }^{*} p<.05 ; * * p<.01 ; * * * p<.001$ 


\section{General Discussion}

We conducted six studies to investigate the extent to which facial feedback effects are moderated by demand characteristics and participants' beliefs. In Studies 1-3, we investigated the impact of demand characteristics by manipulating what participants were told about our hypothesis. With data collected in the U.S. and Kenya, we found that demand characteristics moderated but did not fully account for observed facial feedback effects. In Studies 4-6, we measured participants' beliefs about facial feedback effects. With data collected in 29 countries, we found that these beliefs moderated but did not fully account for observed facial feedback effects.

Notably, our results indicate that participants exhibit facial feedback effects even when they are explicitly told or personally believe that facial poses do not influence emotions. This is notable given that these are conditions in which demand and placebo effects are predicted to work against potential facial feedback effects. According to Mayo's (1991) severe testing philosophy, the strength of evidence should be considered a function of the probability of falsely yielding hypothesis-consistent results. If the facial feedback hypothesis is false, researchers are less likely to erroneously observe the effect in a context where demand and placebo effects work against the facial feedback hypothesis (vs. are minimized, as done in previous research). Thus, our results provide the strongest evidence to date that facial feedback can exert non-artifactual effects on emotional experience. Furthermore, the cultural diversity of our samples bolsters confidence in the cross-cultural generalizability of facial feedback effects. 


\section{Implications for the facial feedback hypothesis}

\section{The role of demand characteristics and participants' beliefs}

Contrary to our initial predictions, our results suggest that demand and placebo effects do not fully drive facial feedback effects. Facial feedback researchers have long been concerned about the role of these study artifacts (e.g., Buck, 1980), but many considered these concerns to be addressed by studies featuring unobtrusive facial feedback manipulations (for a review, see McIntosh, 1996). For example, Strack, Martin, and Stepper (1988) found that participants rated cartoons as more humorous when holding a pen in their mouth in a manner that facilitated vs. inhibited smiling. These participants were apparently unaware that the task impacted their facial expressions, making it unlikely that they correctly inferred the purpose of the study (leading to demand effects) or considered their own beliefs about facial feedback (leading to placebo effects). However, subsequent large-scale collaborations (Coles et al., 2021; Wagenmakers et al., 2016) and a meta-analysis (Coles, Larsen, \& Lench, 2019) have not provided clear evidence that unobtrusive facial feedback manipulations impact emotion. These findings raise the possibility that the effects of overt facial poses on emotion reports are nothing more than study artifacts. Contrary to this view, our results indicated that overt facial feedback manipulations impact emotion even when participants are explicitly told or personally believed that these poses do not influence emotions.

Although our observed facial feedback effects do not appear to be fully driven by demand or placebo effects, our results simultaneously indicate that these methodological artifacts can moderate facial-feedback effects. Indeed, participants exhibited larger facial feedback effects when they were told that the experimenter wanted (vs. did not want) these effects to emerge; participants similarly exhibited larger facial feedback effects when they personally believed vs. 
did not believe in these effects. Hence, researchers should remain cognizant of demand and placebo effects, especially given that a sizeable proportion of our participants had pre-existing beliefs about facial feedback.

Researchers investigating other phenomena should similarly be cognizant of the role of these study artifacts. After all, demand characteristics can be present in any study and participants can have pre-existing beliefs about a variety of psychological phenomena. Observing that methodological artifacts did not drive facial feedback effects in our experimental paradigms does not mean they do not drive other effects in other contexts. For example, some individuals may possess lay beliefs about the motivational benefits of "game faces": posed expressions of seriousness, determination, or anger (Richesin, Oliver, Baldwin, \& Wicks, 2020). These preexisting beliefs could create placebo effects that cause researchers to erroneously observe, for example, that participants are more motivated to compete when posing angry vs. sad expressions. Our evidence of the role of demand and placebo effects is perhaps most important for ongoing discussions about whether blocking frowning facial feedback via botulinum toxin (e.g., via Botox) decreases depression (Alam, Barrett, Hodapp, \& Arndt, 2008; Alves, Sobreira, Aleixo, \& Oliveira, 2016; Chugh, Chhabria, Jung, Kruger, \& Wollmer, 2018; Finzi, 2018; Finzi \& Rosenthal, 2014; Finzi \& Wasserman, 2006; Fromage, 2018; Han et al., 2012; Hexsel et al., 2013; Lewis, 2018; Lewis \& Bowler, 2009; Magid, Finzi, et al., 2015; Magid, Keeling, \& Reichenberg, 2015; Magid et al., 2014; Parsaik et al., 2016; Reichenberg et al., 2016; Wollmer et al., 2012; Wollmer, Kalak, et al., 2014; Wollmer, Magid, \& Kruger, 2014; Zamanian, Jolfaei, Mehran, \& Azizian, 2017). Many studies examining the effects of botulinum toxin on depression are purported to be placebo-controlled because they contain a control group that receives a saline injection (Brin et al., 2020; Finzi \& Rosenthal, 2014; Magid et al., 2014; Wollmer et al., 2012; 
Zamanian et al., 2017). However, because saline injections do not paralyze facial muscles (as does botulinum toxin), participants may easily guess their assigned condition. Thus, researchers have speculated that the effects of botulinum toxin on depression may be driven by placebo effects (Coles \& Larsen, 2021; Coles, Larsen, Kuribayashi, et al., 2019; Rudorfer, 2018). Our results bolster this argument. Given how impactful participants' beliefs and demand characteristics were in our relatively short-term investigation of facial feedback, it is possible their impact is even more pronounced when considering the long-term therapeutic effects of a non-blinded facial feedback intervention (Funder \& Ozer, 2019).

\section{Semantic-link priming: Another artifact in facial feedback research?}

An anonymous reviewer identified another potential artifact in facial feedback studies: semantic priming. Applied to the present studies, our demand characteristics manipulations and facial feedback belief questionnaires often made semantic links between facial expressions and emotional states salient. For example, in the Study 1 nil-hypothesis condition, participants were told “...smiling may make you happier and scowling may make you angrier... We believe this is not true" (emphasis added). Independent of demand effects, instructions like this may activate semantic links between concepts like "smiling" and "happiness". Based solely on the salient semantic links, participants may subsequently report higher levels of happiness after smiling.

At first glance, the semantic-priming artifact account appears similar to a mechanism that many researchers posit underlies facial feedback effects: changes in emotion-related cognitive processes (see Figure 1). For example, some theorists posit that facial feedback impacts emotion because it primes associated memories, thoughts, and concepts (for a review, see Niedenthal, 2007). This differs from the semantic-priming account, however, in its proposed mechanism. The theory-relevant explanation posits that emotion-related concepts are activated by the physical act 
of moving one's face, perhaps due to innate or learned connections with emotion concepts. The semantic-priming artifact account, on the other hand, posits that emotion-related concepts are activated by the processing of semantic information (e.g., hearing or seeing the word "smile" vs. actually posing a smile).

Theoretical nuance aside, it is clear that our observed facial feedback effects cannot be fully explained by semantic-priming artifacts. For example, semantic links were indeed introduced in the instructions presented in the positive- and nil-hypothesis conditions. However, they were not introduced in the no-hypothesis condition, which did not include instructions that referenced facial movements or emotion. Here there were no primed semantic links between facial movements and emotions, yet there was evidence of facial feedback effects. Furthermore, the Study 6 manipulation of whether beliefs were measured before vs. after the study provides a direct test of the impact of primed semantic links. The belief measure certainly primes semantic links, but only half the participants completed the measure before engaging in the facial feedback tasks. If semantic links are responsible for facial feedback effects, we should have observed Pose by Belief Timing interactions, wherein the effects of posed expressions were only significant when facial feedback beliefs were measured at the beginning of the study. We did not find evidence of these interactions - and we observed similar patterns of results regardless of when we measured beliefs about facial feedback. Consequently, concerns about semantic-link artifacts, like demand and placebo effects, cannot fully account for our observed facial feedback effects. 


\section{Characterizing genuine facial feedback effects}

Now that we have provided a better understanding of the role of methodological artifacts, the next step for future researchers is to investigate the mechanisms that drive genuine facial feedback effects, such as those depicted in Figure 1. To that end, three aspects of our findings are useful to emphasize.

First, although researchers often examine whether facial feedback can modulate ongoing emotional responses to things like cartoons (e.g., Strack et al., 1988), our results indicate that facial feedback can also initiate emotional reactions (e.g., in the absence of emotional stimuli). These results converge with recent meta-analytic evidence that facial feedback can both modulate and initiate emotional experiences (Coles, Larsen, \& Lench, 2019). Initiation effects are interesting because many early theorists posited that facial feedback can only modulate ongoing emotional episodes. For example, Allport (1922, 1924) suggested that emotionallyevocative events lead to diffuse feelings of positive and negative affect that are subsequently mentally categorized into discrete emotion categories based on facial feedback. Based on this view, facial feedback cannot initiate, for example, feelings of anger in otherwise neutral contexts_-but it can help determine whether an ongoing negative affective state is mentally categorized as anger (vs. sadness). Our participants engaged in facial poses in relatively neutral contexts and reported coinciding feelings of happiness and anger. These observed patterns are starkly inconsistent with Allport's seminal theory, and they indicate that facial feedback has more than just a modulating effect on emotion.

Our observation that facial feedback can initiate emotional experiences is also interesting from the perspective of appraisal theories of emotion, which emphasize the role of cognitive appraisals (not posed facial expressions) in producing emotions (Moors, Ellsworth, Scherer, \& 
Frijda, 2013). One way that appraisal theories can accommodate our findings is to acknowledge that cognitive appraisals are the primary—but not only_antecedent of emotional experience (Ellsworth \& Scherer, 2003). Alternatively, it is possible that cognitive appraisals are indeed the sole antecedents of emotional experience, and that the effects of facial feedback on emotion are mediated by changes in appraisals (e.g., situations being evaluated more positively when posing smiles; Smith \& Kirby, 2004). However, this would present a somewhat unusual explanation for our findings. For example, many appraisal theories suggest that people will experience anger when they believe that someone else is to blame for a negative outcome (Smith \& Ellsworth, 1985), but it is not clear how our relatively bland experimental procedures could have been evaluated this way.

Second, our results provide preliminary evidence that facial feedback effects may occur non-consciously. Several participants indicated they did not believe in facial feedback effects despite exhibiting such effects mere minutes beforehand. One explanation for the discrepancy between participants' beliefs and their actual emotional responses is that the facial feedback effects may have occurred non-consciously. This, of course, does not suggest that emotions are unconscious - but rather that there may be situations where participants are unaware of the cause of their emotion (Nisbett \& Wilson, 1977). One way to investigate this further would be to simply ask participants after each facial pose trial whether their facial pose affected their emotions. Another approach would be to ask participants whether they noticed any changes in their emotions between facial posing trials (Anvari \& Lakens, 2021). ${ }^{7}$

${ }^{7}$ Although our results provide preliminary evidence that facial feedback effects can occur nonconsciously, we acknowledge that debates about the extent to which any psychological process operates non-consciously are often difficult to resolve. (E.g., for a glimpse of this debate in the 
Third, our results suggest that participants do not need to pose prototypical expressions of emotion to elicit facial feedback effects. Our facial feedback manipulations reliably impacted emotional experience despite being designed to lead to partial re-creations of prototypical expressions of happiness and anger. For example, a prototypical expression of happiness involves activity in both the zygomaticus major and orbicularis oculi muscles (Ekman, Davidson, \& Friesen, 1990), but our smiling manipulation was only designed to produce activity in the zygomaticus major muscles. These results challenge claims that facial feedback effects only occur if the pose creates a prototypical expression of emotion (i.e., an all-or-nothing facial feedback effect; Hager \& Ekman, 1981; Matsumoto, 1987; Tomkins, 1981). Nonetheless facial feedback effects may have been larger if participants had posed more prototypical expressions of emotion (Cross, Gheorma, \& Pressman, 2019; Soussignan, 2002). Future research can investigate this possibility by manipulating the extent to which facial poses match prototypical expressions of emotion.

One issue that our data do not allow us to weigh in on is whether facial feedback impacts (a) more general feelings of valence (i.e., positivity and negativity) and arousal, vs. (b) more specific feelings of discrete emotions (e.g., anger and sadness). We did not, for example, include multiple same-valence facial feedback manipulations, and we did not measure general feelings of positivity and negativity. Nonetheless, we will note that exploratory analyses did not provide consistent evidence of valence-specific facial feedback effects. That is, we did not reliably observe that posed angry expressions decreased feelings of happiness or increased feelings of fear (see Supplemental Information).

evaluative conditioning literature, see Hütter, Sweldens, Stahl, Unkelbach, \& Klauer, 2012; Kattner, 2012; March, Olson, \& Fazio, 2018; Mitchell, De Houwer, \& Lovibond, 2009; Pleyers, Corneille, Luminet, \& Yzerbyt, 2007). 


\section{Implications for research on methodological artifacts}

Our finding that demand characteristics can moderate facial feedback effects is inconsistent with recent work by Mummolo and Peterson (2019), who found little-to-no evidence that a variety of demand characteristic manipulations impacted participants' responses in online replications of classic behavioral economic studies. For example, in one study, participants were asked to indicate their willingness to call a hypothetical job applicant who was randomlyassigned a stereotypical European American or African American name. Before reviewing the resume, participants were informed that the experimenter expected them to prefer either European American or African American candidates. Contrary to concerns about demand effects, the hypothesis communicated did not affect the probability that participants called candidates with European American and African American names. Based on findings like this, many researchers have questioned the impact of demand characteristics, arguing that demand effects are "rare" (Clayton et al., 2020, p. 1092), supported by "little evidence" (Guess \& Coppock, 2020, p. 1512), and/or "limited" (Vial, Brescoll, \& Dovidio, 2019, p. 94). Our own observations of demand effects suggest that such conclusions are premature.

How can we explain the discrepancy between our findings and those of Mummolo and Peterson (2019)? One challenge is that there has been little formal analysis of how and when demand characteristics impact behavior. Fortunately, Corneille and Lush (2021) have recently developed a more extensive model of demand characteristics. According to their model, demand characteristics can indeed cause participants to change their behavior to help the experimenter confirm a hypothesis (the mechanism that most researchers assume underlie the effects of demand characteristics; see Strohmetz, 2008). However, their model also suggests that demand characteristics can activate other goals, such as the goal to respond in a socially desirable 
manner. Furthermore, these goals can sometimes come into conflict (for discussions of goal conflict, see Boudreaux \& Ozer, 2013). In Mummolo and Peterson's experiments, participants were often presented with hypothesized patterns of behavior that may have run counter to their own values and subjective norms. For example, in the resume experiment, participants' motivation to help the experimenters confirm their hypothesis may have been eclipsed by their motivation to avoid appearing biased (for similar findings, see Sigall et al., 1970). In contrast, it is unlikely that anyone's beliefs about the effects of facial expressions on emotions are wrapped up in their core values. Thus, by limiting the role of competing motivations, our work may have maximized the impact of participants' motivation to help us confirm our hypotheses.

\section{Conclusion}

Researchers' views about the facial feedback hypothesis have drastically fluctuated in recent years, and our results call for further updating. Less than a decade ago, nearly all narrative reviews concluded that facial feedback can influence emotional experience (Adelmann \& Zajonc, 1989; Cappella, 1993; Izard, 1990; Laird, 1984; Laird \& Lacasse, 2014; Martin, 1990; McIntosh, 1996; Price \& Harmon-Jones, 2015; Whissell, 1985), and the facial feedback hypothesis was a literal textbook phenomenon. However, in the midst of a replication crisis (Pashler \& Wagenmakers, 2012) and the aftermath of many labs' failure to replicate a seminal demonstration of facial feedback effects (Wagenmakers et al., 2016), researchers changed their tune. For example, in the wake of Wagenmakers et al.'s (2016) failure to replicate, Steinmetz and Posten (2017) said that the evidence for facial feedback effects is "inconclusive" (p. 288); Smith and Apicella (2017) argued that such effects have "not survived replication" (p. 179); and Nicholas and Ashton-James (2017) concluded the effects are "at best, very small and difficult to replicate, and at worst... a by-product of methodological confounds" (p. 994, emphasis added). 
We initially shared this pessimistic view, but have since updated our beliefs in light of evidence of facial feedback effects from a comprehensive meta-analysis (Coles, Larsen, \& Lench, 2019), a multi-country registered report (Coles et al., 2021), and (even more recently) the present work. Indeed, the present work not only demonstrates that facial feedback effects can be replicablebut also clearly differentiated from methodological artifacts such as demand and placebo effects. 


\section{Author Contributions}

N. A. Coles developed the initial study concept. N. A. Coles, L. Gaertner, B. Frohlich, J. Larsen, and D. Basnight-Brown developed the methodology. N. A. Coles and B. Frohlich programmed the study. N. A. Coles, B. Frohlich, J. Larsen, and D. Basnight-Brown supervised data collection. N. A. Coles and L. Gaertner analyzed and visualized the data. N. A. Coles drafted the manuscript. L. Gaertner, B. Frohlich, J. T. Larsen, and D. Basnight-Brown provided critical feedback on the manuscript. All authors contributed to revisions and approved the final version of the manuscript for submission.

\section{Acknowledgments}

Nicholas A. Coles was supported by the National Science Foundation Graduate Research Fellowship \#R010138018. This work was also financially supported by Dr. Brad Stastny, who generously donated funds for this research in memory of his father, Bill Stastny. Thank you to

Åse Innes-Ker, Nicholas James, Tiago Lubiana, Melissa Kline, Ingrid Aulike, Pradnya Bulusu, and Jonathan Williams for their earlier feedback through the Red Team Challenge: a financially incentivized error-detection challenge. Thank you to Leo Tiokhin, Daniël Lakens, and Ruben Arslan for comments on an earlier version of this manuscript. Study 1 was completed for B. Frohlich's undergraduate thesis, which was completed in the Department of Psychology at the University of Tennessee. 


\section{References}

Adelmann, P. K., \& Zajonc, R. B. (1989). Facial efference and the experience of emotion. Annual Review of Psychology, 40, 249-280.

Alam, M., Barrett, K. C., Hodapp, R. M., \& Arndt, K. A. (2008). Botulinum toxin and the facial feedback hypothesis: Can looking better make you feel happier? Journal of the American Academy of Dermatology, 58(6), 1061-1072.

Allport, F. H. (1922). A physiological-genetic theory of feeling and emotion. Psychological Review, 29(2), 132-139.

Allport, F. H. (1924). Feeling and emotion. In F. H. Allport (Ed.), Social Psychology (pp. 8498). Boston: Houghton Mifflin Company.

Altman, D. G., \& Royston, P. (2006). The cost of dichotomising continuous variables. British Medical Journal, 332(7549), 1080.

Alves, M. C., Sobreira, G., Aleixo, M. A., \& Oliveira, J. M. (2016). Facing depression with botulinum toxin: Literature review. European Psychiatry, 335, 5290-5643.

Anvari, F., \& Lakens, D. (2021). Using anchor-based methods to determine the smallest effect size of interest. Journal of Experimental Social Psychology, 96.

Arnone, D., Galadari, H., Rodgers, C. J., Östlundh, L., Aziz, K. A., Stip, E., \& Young, A. H. (2021). Efficacy of onabotulinumtoxinA in the treatment of unipolar major depression: Systematic review, meta-analysis and meta-regression analyses of double-blind randomised controlled trials. Journal of Psychopharmacology.

Boudreaux, M. J., \& Ozer, D. J. (2013). Goal conflict, goal striving, and psychological wellbeing. Motivation and Emotion, 37(3), 433-443.

Brin, M. F., Durgam, S., Lum, A., James, L., Liu, J., Thase, M. E., \& Szegedi, A. (2020). 
OnabotulinumtoxinA for the treatment of major depressive disorder: a phase 2 randomized, double-blind, placebo-controlled trial in adult females. International Clinical Psychopharmacology, 35(1), 19-28.

Buck, R. (1980). Nonverbal behavior and the theory of emotion: The facial feedback hypothesis. Journal of Personality and Social Psychology, 38(5), 811-824.

Cacioppo, J. T., Berntson, G. G., \& Klein, D. J. (1992). What is an emotion? The role of somatovisceral afference, with special emphasis on somatovisceral "illusions." In M. S. Clark (Ed.), Review of personality and social psychology: Emotion and social behavior (pp. 63-98). Sage Publications, Inc.

Cappella, J. N. (1993). The facial feedback hypothesis in human interaction: Review and speculation. Journal of Language and Social Psychology, 12, 13-29.

Carpenter, S. M., \& Niedenthal, P. M. (2020). Disrupting facial action increases risk taking. Emotion, 20(6), 1084-1092.

Chugh, S., Chhabria, A., Jung, S., Kruger, T. H. C., \& Wollmer, M. A. (2018). Botulinum toxin as a treatment for depression in a real-world setting. Journal of Psychiatric Practice, 24(1), $15-20$.

Clayton, K., Blair, S., Busam, J. A., Forstner, S., Glance, J., Green, G., ... Nyhan, B. (2020). Real solutions for fake news? Measuring the effectiveness of general warnings and factcheck tags in reducing belief in false stories on social media. Political Behavior, 42(4), 1073-1095.

Coan, J. A., Allen, J. J. B., \& Harmon-Jones, E. (2001). Voluntary facial expression and hemispheric asymmetry over the frontal cortex. Psychophysiology, 38(6), 912-925.

Coles, N. A. (2020). Face and feeling: An examination of the role of facial feedback in emotion. 
Retrieved from https://trace.tennessee.edu/utk_graddiss/5874

Coles, N. A., \& Larsen, J. T. (2021). Letter to the Editor: Claims about the effects of botulinum toxin on depression should raise some eyebrows. Journal of Psychiatric Research, 140, $551-552$.

Coles, N. A., Larsen, J. T., Kuribayashi, J., \& Kuelz, A. (2019). Does blocking facial feedback via botulinum toxin injections decrease depression? A critical review and meta-analysis. Emotion Review, 11(4), 294-309.

Coles, N. A., Larsen, J. T., \& Lench, H. C. (2019). A meta-analysis of the facial feedback literature: Effects of facial feedback on emotional experience are small and variable. Psychological Bulletin, 145(6), 610-651.

Coles, N. A., March, D. S., Marmolejo-Ramos, F., Banaruee, H., Butcher, N., Cavallet, M., ... Gorbunova, E. (2021). A multi-lab test of the facial feedback hypothesis by The Many Smiles Collaboration. Retrieved from https://psyarxiv.com/cvpuw

Corneille, O., \& Lush, P. (2021). Sixty years after Orne's American Psychologist article: A conceptual analysis of "Demand Characteristics". 1-70. Retrieved from https://psyarxiv.com/jqyvx/

Cross, M. P., Gheorma, L., \& Pressman, S. D. (2019). Contrasting experimentally devicemanipulated and device-free smiles. Frontiers in Psychology, 10(2297), 1-11.

Damasio, A., \& Carvalho, G. B. (2013). The nature of feelings: Evolutionary and neurobiological origins. Nature Reviews Neuroscience, 14(2), 143-152.

de Quidt, J., Haushofer, J., \& Roth, C. (2018). Measure and bounding experimenter demand. American Economic Review, 108(11), 3266-3302.

Dimberg, U., \& Söderkvist, S. (2011). The voluntary facial action technique: A method to test 
the facial feedback hypothesis. Journal of Nonverbal Behavior, 35(1), 17-33.

Ekman, P., Davidson, R. J., \& Friesen, W. V. (1990). The Duchenne Smile: Emotional expression and brain physiology II. Journal of Personality and Social Psychology, 58(2), $342-353$.

Ekman, P., Levenson, R. W., \& Friesen, W. V. (1983). Autonomic nervous system activity distinguishes among emotions. Science, 221(4616), 1208-1210.

Ellsworth, P. C., \& Scherer, K. R. (2003). Appraisal Processes in Emotion. Handbook of Affective Sciences.

Finzi, E. (2018). Update: Botulinum toxin for depression: More than skin deep. Dermatologic Surgery, 44(10), 1363-1365.

Finzi, E., \& Rosenthal, N. E. (2014). Treatment of depression with onabotulinumtoxinA: A randomized, double-blind, placebo controlled trial. Journal of Psychiatric Research, 52, 16.

Finzi, E., \& Wasserman, E. (2006). Treatment of depression with botulinum toxin A: A case series. Dermatologic Surgery, 32(5), 645-649.

Flack, W. (2006). Peripheral feedback effects of facial expressions, bodily postures, and vocal expressions on emotional feelings. Cognition \& Emotion, 20(2), 177-195.

Förster, J., \& Strack, F. (1996). Influence of overt head movements on memory for valenced words: A case of conceptual-motor compatibility. Journal of Personality and Social Psychology, 71(3), 421-430.

Fromage, G. (2018). Exploring the effects of botulinum toxin type A injections on depression. Aesthetic Nursing, 7(6), 315-317.

Funder, D. C., \& Ozer, D. J. (2019). Evaluating effect size in psychological research: Sense and 
nonsense. Advances in Methods and Practices in Psychological Science, 2(2), 156-168.

Guess, A., \& Coppock, A. (2020). Does counter-attitudinal information cause backlash? Results from three large survey experiments. British Journal of Political Science, 50(4), 14971515.

Gustafson, L. A., \& Orne, M. T. (1965). Effects of perceived role and role success on the detection of deception. Journal of Applied Psychology, 49(6), 412.

Hager, J. C., \& Ekman, P. (1981). Methodological problems in Tourangeau and Ellsworth's study of facial expression and experience of emotion. Journal of Personality and Social Psychology, 40(2), 358-362.

Han, C., Park, G.-Y., Wang, S.-M., Lee, S.-Y., Lee, S.-J., Bahk, W.-M., \& Pae, C.-U. (2012). Can botulinum toxin improve mood in depressed patients? Expert Review of Neurotherapeutics, 12(9), 1049-1051.

Harmon-Jones, C., Bastian, B., \& Harmon-Jones, E. (2016). The discrete emotions questionnaire: A new tool for measuring state self-reported emotions. PloS One, 11(8), e0159915.

Harmon-Jones, E., Gable, P. A., \& Price, T. F. (2011). Leaning embodies desire: Evidence that leaning forward increases relative left frontal cortical activation to appetitive stimuli. Biological Psychology, 87(2), 311-313.

Hatfield, E., Cacioppo, J. T., \& Rapson, R. L. (1992). Primitive emotional contagion. In M. S. Clark (Ed.), Review of personality and social psychology: Vol. 14: Emotions and social behavior (pp. 151-177). Newbury Park, CA: Sage.

Henrich, J., Heine, S. J., \& Norenzayan, A. (2010). The weirdest people in the world? Behavioral and Brain Sciences, 33(2-3), 61-83. 
Hexsel, D., Brum, C., Siega, C., Schilling-Souza, J., Dal'Forno, T., Heckmann, M., \& Rodrigues, T. C. (2013). Evaluation of self-esteem and depression symptoms in depressed and nondepressed subjects treated with onabotulinumtoxinA for glabellar lines. Dermatologic Surgery, 39(7), 1088-1096.

Hoffman, M. L. (2001). Empathy and moral development: Implications for caring and justice. Cambridge University Press.

Holland, A. C., Connell, G. O., \& Dziobek, I. (2020). Facial mimicry, empathy, and emotion recognition: a meta-analysis of correlations. Cognition and Emotion, 1-19.

Hütter, M., Sweldens, S., Stahl, C., Unkelbach, C., \& Klauer, K. C. (2012). Dissociating contingency awareness and conditioned attitudes: Evidence of contingency-unaware evaluative conditioning. Journal of Experimental Psychology: General, 141(3), 539.

Izard, C. E. (1979). Human Emotions. (C. E. Izard \& J. L. Singer, Eds.). New York: Plenum Publishing Corporation.

Izard, C. E. (1990). Facial expressions and the regulation of emotions. Journal of Personality and Social Psychology, 58(3), 487-498.

Izard, C. E. (2007). Basic emotions, natural kinds, emotion schemas, and a new paradigm. Perspectives on Psychological Science, 2(3), 260-280.

Kaiser, J., \& Davey, G. C. L. (2017). The effect of facial feedback on the evaluation of statements describing everyday situations and the role of awareness. Consciousness and Cognition, 53, 23-30.

Kattner, F. (2012). Revisiting the relation between contingency awareness and attention: Evaluative conditioning relies on a contingency focus. Cognition and Emotion, 26(1), 166175. 
Kersbergen, I., Whitelock, V., Haynes, A., Schroor, M., \& Robinson, E. (2019). Hypothesis awareness as a demand characteristic in laboratory-based eating behaviour research: An experimental study. Appetite, 141(June), 104318.

Kirsch, I. (1997). Response expectancy theory and application: A decennial review. Applied and Preventive Psychology, 6(2), 69-79.

Kirsch, I. (2018). Response expectancy and the placebo effect. International Review of Neurobiology, 138, 81-93.

Kraft, T. L., \& Pressman, S. D. (2012). Grin and bear it: The influence of manipulated facial expression on the stress response. Psychological Science, 23(11), 1372-1378.

Laird, J. D. (1974). Self-attribution of emotion: The effects of expressive behavior on the quality of emotional experience. Journal of Personality and Social Psychology, 29(4), 475-486.

Laird, J. D. (1984). The real role of facial response in the experience of emotion: A reply to Tourangeau and Ellsworth, and others. Journal of Personality and Social Psychology, 47(4), 909-917.

Laird, J. D., \& Bresler, C. (1992). The process of emotional experience: A self-perception theory. In M. S. Clark (Ed.), Review of Personality and Social Psychology: Emotion (pp. 213-234). Newbury Park.

Laird, J. D., \& Lacasse, K. (2014). Bodily influences on emotional feelings: Accumulating evidence and extensions of William James's theory of emotion. Emotion Review, 6(1), 2734.

Larsen, J. T., \& McGraw, a P. (2011). Further evidence for mixed emotions. Journal of Personality and Social Psychology, 100(6), 1095-1110.

Larsen, R. J., Kasimatis, M., \& Frey, K. (1992). Facilitating the furrowed brow: An unobtrusive 
test of the facial feedback hypothesis applied to unpleasant affect. Cognition and Emotion, 6(5), 321-338.

Levenson, R. W., Carstensen, L. L., Friesen, W. V, \& Ekman, P. (1991). Emotion, physiology, and expression in old age. Psychology and Aging, 6(1), 28-35.

Levenson, R. W., Ekman, P., \& Friesen, W. V. (1990). Voluntary facial action generates emotion-specific autonomic nervous system activity. Psychophysiology, 27(4), 363-384.

Levenson, R. W., Ekman, P., Heider, K., \& Friesen, W. V. (1992). Emotion and autonomic nervous system activity in the Minangkabau of West Sumatra. Journal of Personality and Social Psychology, 62(6), 972-988.

Lewinski, P., den Uyl, T. M., \& Butler, C. (2014). Automated facial coding: Validation of basic emotions and FACS AUs in FaceReader. Journal of Neuroscience, Psychology, and Economics, 7(4), 227-236.

Lewis, M. B. (2018). The interactions between botulinum-toxin-based facial treatments and embodied emotions. Scientific Reports, 8(1), 1-10.

Lewis, M. B., \& Bowler, P. J. (2009). Botulinum toxin cosmetic therapy correlates with a more positive mood. Journal of Cosmetic Dermatology, 8(1), 24-26.

Magid, M., Finzi, E., Kruger, T. H. C., Robertson, H. T., Keeling, B. H., Jung, S., ... Wollmer, M. A. (2015). Treating depression with botulinum toxin: A pooled analysis of randomized controlled trials. Pharmacopsychiatry, 48(6), 205-210.

Magid, M., Keeling, B. H., \& Reichenberg, J. S. (2015). Neurotoxins: Expanding uses of neuromodulators in medicine-Major Depressive Disorder. Plastic and Reconstructive Surgery, 136, 111S-119S.

Magid, M., Reichenberg, J. S., Poth, P. E., Robertson, H. T., LaViolette, A. K., Kruger, T. H. C., 
\& Wollmer, M. A. (2014). Treatment of major depressive disorder using botulinum toxin A: A 24-week randomized, double-blind, placebo-controlled study. Journal of Clinical Psychiatry, 75(8), 837-844.

March, D. S., Olson, M. A., \& Fazio, R. H. (2018). The implicit misattribution model of evaluative conditioning. Social Psychological Bulletin, 13(3), 1-25.

Marmolejo-Ramos, F., Murata, A., Sasaki, K., Yamada, Y., Ikeda, A., Hinojosa, J. A., ... Ospina, R. (2020). Your face and moves seem happier when I smile: Facial action influences the perception of emotional faces and biological motion stimuli. Experimental Psychology, 32(17), 1-27.

Marsh, A. A., Rhoads, S. A., \& Ryan, R. M. (2019). A multi-semester classroom demonstration yields evidence in support of the facial feedback effect. Emotion, 19(8), 1500-1504.

Martin, M. (1990). On the induction of mood. Clinical Psychology Review, 10(6), 669-697.

Matsumoto, D. (1987). The role of facial response in the experience of emotion: More methodological problems and a meta-analysis. Journal of Personality and Social Psychology, 52(4), 769-774.

Mayo, D. G. (1991). Novel evidence and severe tests. Philosophy of Science, 58(4), 523-552.

McIntosh, D. N. (1996). Facial feedback hypotheses: Evidence, implications, and directions. Motivation and Emotion, 20(2), 121-147.

Mehling, W. E., Price, C., Daubenmier, J. J., Acree, M., Bartmess, E., \& Stewart, A. (2012). The multidimensional assessment of interoceptive awareness (MAIA). PLOS ONE, 7(11), 1-22.

Mitchell, C. J., De Houwer, J., \& Lovibond, P. F. (2009). The propositional nature of human associative learning. Behavioral and Brain Sciences, 32(2), 183-198.

Moors, A., Ellsworth, P. C., Scherer, K. R., \& Frijda, N. H. (2013). Appraisal theories of 
emotion: State of the art and future development. Emotion Review, 5(2), 119-124.

Mummolo, J., \& Peterson, E. (2019). Demand effects in survey experiments: An empirical assessment. American Political Science Review, 113(2), 517-529.

Neal, D. T., \& Chartrand, T. L. (2011). Embodied emotion perception: Amplifying and dampening facial feedback modulates emotion perception accuracy. Social Psychological and Personality Science, 2(6), 673-678.

Nicholas, M. K., \& Ashton-James, C. (2017). Embodied pain: Grasping a thorny problem? Pain, 158(6), 993-994.

Nichols, A. L., \& Maner, J. K. (2008). The good-subject effect: Investigating participant demand characteristics. Journal of General Psychology, 135(2), 151-166.

Niedenthal, P. M. (2007). Embodying emotion. Science, 316(5827), 1002-1005.

Niedenthal, P. M., Barsalou, Lawrence Winkielman, P., Krauth-Gruber, S., \& Ric, F. (2005).

Embodiment in attitudes, social perception, and emotion. Personality and Social Psychology Review, 9(3), 184-211.

Niedenthal, P. M., Barsalou, L. W., Rig, F., \& Krauth-Gruber, S. (2005). Embodiment in the acquisition and use of emotion knowledge. In L. Feldman Barrett, P. M. Niedenthal, \& P. Winkielman (Eds.), Emotion: Conscious and Unconscious (pp. 21-50). Guilford Press.

Niedenthal, P. M., Mermillod, M., Maringer, M., \& Hess, U. (2010). The Simulation of Smiles (SIMS) model: Embodied simulation and the meaning of facial expression. Behavioral and Brain Sciences, 33, 417-480.

Niedenthal, P. M., Winkielman, P., Mondillon, L., \& Vermeulen, N. (2009). Embodiment of emotion concepts. Journal of Personality and Social Psychology, 96(6), 1120-1136.

Nisbett, R. E., \& Wilson, T. D. (1977). Telling more than we can know: Verbal reports on 
mental processes. Psychological Review, 84(3), 231-259.

Noah, T., Schul, Y., \& Mayo, R. (2018). When both the original study and its failed replication are correct: Feeling observed eliminates the facial-feedback effect. Journal of Personality \& Social Psychology, 114(5), 1-26.

Orne, M. T. (1959). The nature of hypnosis: Artifact and essence. The Journal of Abnormal and Social Psychology, 58(3), 277-299.

Orne, M. T. (1962). On the social psychology of the psychological experiment. American Psychologist, 17(11), 776-783.

Parsaik, A. K., Mascharenhas, S. S., Hashmi, A., Prokop, L. J., John, V., Okusaga, O., \& Singh, B. (2016). Role of botulinum toxin in depression. Journal of Psychiatric Practice, 22(2), 99-110.

Pashler, H., \& Wagenmakers, E.-J. (2012). Editors' introduction to the special section on replicability in psychological science: A crisis of confidence? Perspectives on Psychological Science, 7(6), 528-530.

Pleyers, G., Corneille, O., Luminet, O., \& Yzerbyt, V. (2007). Aware and (dis)liking: Item-based analyses reveal that valence acquisition via evaluative conditioning emerges only when there is contingency awareness. Journal of Experimental Psychology: Learning, Memory, and Cognition, 33(1), 130.

Price, T. F., \& Harmon-Jones, E. (2015). Embodied emotion: The influence of manipulated facial and bodily states on emotive responses. Wiley Interdisciplinary Reviews. Cognitive Science, 6(6), 461-473.

Reichenberg, J. S., Hauptman, A. J., Robertson, H. T., Finzi, E., Kruger, T. H. C., Wollmer, M. A., \& Magid, M. (2016). Botulinum toxin for depression: Does patient appearance matter? 
Journal of the American Academy of Dermatology, 74(1), 171-173.

Richesin, M. T., Oliver, M. D., Baldwin, D. R., \& Wicks, L. A. M. (2020). Game Face expressions and performance on competitive tasks. Stress and Health, 36(2), 166-171.

Rinn, W. E. (1984). The neuropsychology of facial expression: A review of the neurological and psychological mechanisms for producing facial expressions. Psychological Bulletin, 95(1), $52-77$.

Roche, B., Forsyth, J. P., \& Maher, E. (2007). The impact of demand characteristics on brief acceptance- and control-based interventions for pain tolerance. Cognitive and Behavioral Practice, 14(4), 381-393.

Rosenthal, R., \& Fode, K. L. (1963). Psychology of the scientist: V. Three experiments in experimenter bias. Psychological Reports, 12, 491-511.

Rudorfer, M. V. (2018). Botulinum toxin: Does it have a place in the management of depression? CNS Drugs, 32, 97-100.

Russell, J. A. (1980). A circumplex model of affect. Journal of Personality and Social Psychology, 39(6), 1161-1178.

Scherer, K. R. (2009). The dynamic architecture of emotion: Evidence for the component process model. Cognition and Emotion, 23(7), 1307-1351.

Scherer, K. R., \& Moors, A. (2019). The emotion process: Event appraisal and component differentiation. Annual Review of Psychology, 70(1), 719-745.

Schielzeth, H., Dingemanse, N. J., Nakagawa, S., Westneat, D. F., Allegue, H., Teplitsky, C., ... Araya-Ajoy, Y. G. (2020). Robustness of linear mixed-effects models to violations of distributional assumptions. Methods in Ecology and Evolution, 11(9), 1141-1152.

Schimmack, U., \& Chen, Y. (2017). The power of the pen paradigm: A replicability analysis. 
Retrieved from https://replicationindex.com/2017/09/04/the-power-of-the-pen-paradigm-areplicability-analysis/

Schnall, S., \& Laird, J. D. (2003). Keep smiling: Enduring effects of facial expressions and postures on emotional experience and memory. Cognition and Emotion, 17(5), 787-797.

Sigall, H., Aronson, E., \& Hoose, T. Van. (1970). The cooperative subject: Myth or reality? Journal of Experimental Social Psychology, 6(1), 1-10.

Skinner, A. L., Osnaya, A., Patel, B., \& Perry, S. P. (2020). Mimicking others' nonverbal signals is associated with increased attitude contagion. Journal of Nonverbal Behavior, 44(1), 117131.

Smith, C. A., \& Ellsworth, P. C. (1985). Patterns of cognitive appraisal in emotion. Journal of Personality and Social Psychology, 48(4), 813-838.

Smith, C. A., \& Kirby, L. D. (2004). Appraisal as a pervasive determinant of anger. Emotion, $4(2), 133-138$.

Smith, K. M., \& Apicella, C. L. (2017). Winners, losers, and posers: The effect of power poses on testosterone and risk-taking following competition. Hormones and Behavior, 92, 172181.

Söderkvist, S., Ohlén, K., \& Dimberg, U. (2018). How the experience of emotion is modulated by facial feedback. Journal of Nonverbal Behavior, 42(1), 129-151.

Soussignan, R. (2002). Duchenne smile, emotional experience, and autonomic reactivity: A test of the facial feedback hypothesis. Emotion, 2(1), 52-74.

Steinmetz, J., \& Posten, A.-C. (2017). Physical temperature affects response behavior. Journal of Experimental Social Psychology, 70, 294-300.

Stewart-Williams, S., \& Podd, J. (2004). The placebo effect: Dissolving the expectancy versus 
conditioning debate. Psychological Bulletin, 130(2), 324-340.

Strack, F., Martin, L. L., \& Stepper, S. (1988). Inhibiting and facilitating conditions of the human smile: A nonobtrusive test of the facial feedback hypothesis. Journal of Personality and Social Psychology, 54(5), 768-777.

Strohmetz, D. B. (2008). Research artifacts and the social psychology of psychological experiments. Social and Personality Psychology Compass, 2(2), 861-877.

Tomkins, S. (1962). Affect Imagery Consciousness: Vol 1 The Positive Affects. New York: Springer.

Tomkins, S. (1981). The role of facial response in the experience of emotion: A reply to Tourangeau and Ellsworth. Journal of Personality and Social Psychology, 37(9), 15191531.

Veitch, J. A., Gifford, R., \& Hine, D. W. (1991). Demand characteristics and full spectrum lighting effects on performance and mood. Journal of Environmental Psychology, 11(1), $87-95$.

Vial, A. C., Brescoll, V. L., \& Dovidio, J. F. (2019). Third-party prejudice accommodation increases gender discrimination. Journal of Personality and Social Psychology, 117(1), 7398.

Wagenmakers, E.-J., Beek, T., Dijkhoff, L., Gronau, Q. F., Acosta, A., Adams, R. B., ... Zwaan, R. A. (2016). Registered replication report: Strack, Martin, \& Stepper (1988). Perspectives on Psychological Science, 11(6), 917-928.

Wansink, B., Zampollo, F., Camps, G., \& Shimizu, M. (2014). Biting versus chewing: Eating style and social aggression in children. Eating Behaviors, 15(2), 311-313.

Weber, S. J., \& Cook, T. D. (1972). Subject effects in laboratory research: An examination of 
subject roles, demand characteristics, and valid inference. Psychological Bulletin, 77(4), 273-295.

Whissell, C. M. (1985). The role of the face in human emotion: First system or one of many? Perceptual and Motor Skills.

Winkielman, P., Coulson, S., \& Niedenthal, P. (2018). Dynamic grounding of emotion concepts. Philosophical Transactions of the Royal Society B: Biological Sciences, 373(1752).

Wollmer, M. A., De Boer, C., Kalak, N., Beck, J., G??tz, T., Schmidt, T., ... Kruger, T. H. C. (2012). Facing depression with botulinum toxin: A randomized controlled trial. Journal of Psychiatric Research, 46(5), 574-581.

Wollmer, M. A., Kalak, N., Jung, S., de Boer, C., Magid, M., Reichenberg, J. S., ... Kruger, T. H. C. (2014). Agitation predicts response of depression to botulinum toxin treatment in a randomized controlled trial. Frontiers in Psychiatry, 5, 1-5.

Wollmer, M. A., Magid, M., \& Kruger, T. H. C. (2014). Botulinum toxin treatment in depression. In A. Bewley, R. E. Taylor, J. S. Reichenberg, \& M. Magid (Eds.), Practical Psychodermatology (pp. 216-219). New Jersey: John Wiley \& Sons.

Wood, A., Rychlowska, M., Korb, S., \& Niedenthal, P. (2016). Fashioning the face: Sensorimotor simulation contributes to facial expression recognition. Trends in Cognitive Sciences, 20(3), 227-240.

Young, S. D., Adelstein, B. D., \& Ellis, S. R. (2006). Demand characteristics of a questionnaire used to assess motion sickness in a virtual environment. Proceedings - IEEE Virtual Reality, 2006, 97-102.

Zamanian, A., Jolfaei, A. G., Mehran, G., \& Azizian, Z. (2017). Efficacy of Botox versus placebo for treatment of patients with major depression. Iranian Journal of Public Health, 
46(7), 982-984. 
FACIAL FEEDBACK AND STUDY ARTIFACTS

Fact or artifact? Demand characteristics and participants' beliefs can moderate, but do not fully account for, the effects of facial feedback on emotional experience

Supplemental Materials 


\section{Study 1 Visual Aid}

Experimenters in Study 1 were permitted to use the visual aids below to explain to participants the expected effects in the positive- and nil-hypothesis conditions.

\section{Figure S1.}

Visual aid used to explain the expected effects in the positive- and nil-hypothesis conditions.

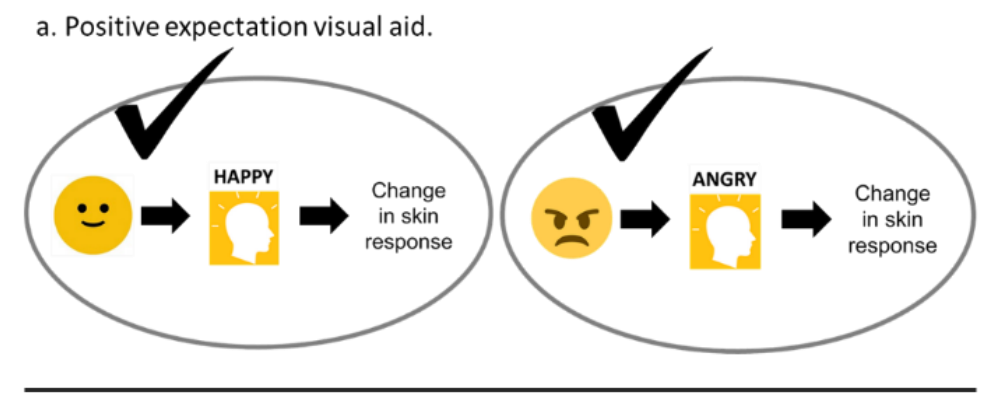

b. Null expectation visual aid.

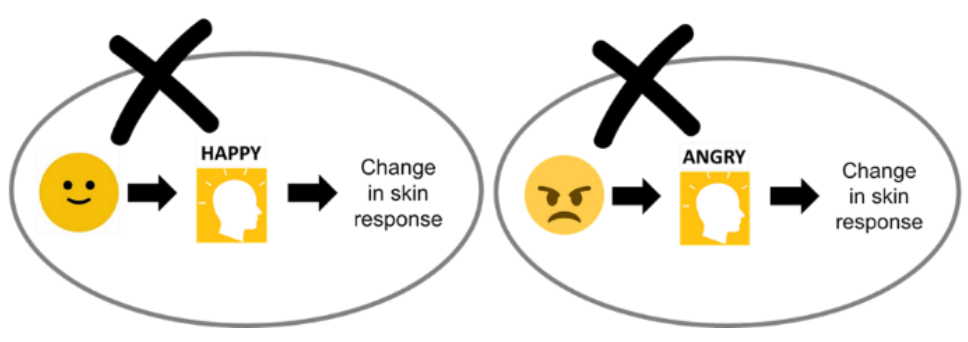

Full ANOVA and pairwise comparison tables

In Studies 1-6, we were primarily interested in testing the Pose by Demand (Studies 1-3) and Pose by Belief (Studies 4-6) interactions. Nevertheless, results from the full ANOVA tables are displayed in Tables S1 and S2. Pairwise comparisons for the decomposition of the interactions are displayed in Tables S3 and S4.

For all tables, we modeled self-reported happiness and anger using linear mixed effects models with Pose, Block, and the study artifact (Demand or Pose) entered as effects-coded factors, all higher-order interactions, random-intercepts for each participant, and Type III Sums of Squares. We used model-estimated marginal means for all pairwise comparisons. 


\section{Table S1.}

Study 1-3 ANOVA table output from linear mixed effects-models of happiness and anger reports. F-values are from ANOVAs with Satterthwaite method degrees of freedom.

\begin{tabular}{|c|c|c|c|c|}
\hline Emotion Report & Parameter & Study 1 & Study 2 & Study 3 \\
\hline \multirow[t]{7}{*}{ Happiness } & Pose & $F(2,1235)=246.03 * * *$ & $F(2,945)=179.42^{* * *}$ & $F(2,645)=81.87 * * *$ \\
\hline & Demand & $F(2,247)=7.34 * * *$ & $F(2,189)=5.04 * *$ & $F(1,129)=1.42$ \\
\hline & Block & $F(1,1235)=19.38 * * *$ & $F(1,945)=10.8 * *$ & $F(1,645)=10.14^{* *}$ \\
\hline & Pose by Demand & $F(4,1235)=24.6^{* * *}$ & $F(4,945)=3.18^{*}$ & $F(2,645)=5.92 * *$ \\
\hline & Pose by Block & $F(2,1235)=4.24^{*}$ & $F(2,945)=1.26$ & $F(2,645)=1.38$ \\
\hline & Demand by Block & $F(2,1235)=0.4$ & $F(2,945)=0.15$ & $F(1,645)=0.06$ \\
\hline & Pose by Demand by Block & $F(4,1235)=0.15$ & $F(4,945)=0.16$ & $F(2,645)=0.41$ \\
\hline \multirow[t]{7}{*}{ Anger } & Pose & $F(2,1235)=146.33 * * *$ & $F(2,945)=108.74 * * *$ & $F(2,645)=104.35^{* * *}$ \\
\hline & Demand & $F(2,247)=9.5^{* * *}$ & $F(2,189)=8.75^{* * *}$ & $F(1,129)=1.77$ \\
\hline & Block & $F(1,1235)=8.3 * *$ & $F(1,945)=0.28$ & $F(1,645)=0$ \\
\hline & Pose by Demand & $F(4,1235)=17.55^{* * *}$ & $F(4,945)=1.58$ & $F(2,645)=10.46^{* * *}$ \\
\hline & Pose by Block & $F(2,1235)=1.13$ & $F(2,945)=0.47$ & $F(2,645)=1.18$ \\
\hline & Demand by Block & $F(2,1235)=0.48$ & $F(2,945)=0.18$ & $F(1,645)=2.06$ \\
\hline & Pose by Demand by Block & $F(4,1235)=1.28$ & $F(4,945)=0.37$ & $F(2,645)=0.81$ \\
\hline
\end{tabular}




\section{Table S2.}

Study 4-6 ANOVA table output from linear mixed effects-models of happiness and anger reports. F-values are from ANOVAs with Satterthwaite method degrees of freedom.

\begin{tabular}{|c|c|c|c|c|}
\hline Emotion & Parameter & Study 4 & Study 5 & Study 6 \\
\hline \multirow[t]{7}{*}{ Happiness } & Pose & $F(2,2715)=170.38 * * *$ & $F(2,375)=85.01 * * *$ & $F(2,1705)=227.94 * * *$ \\
\hline & Belief & $F(2,543)=21.39 * * *$ & $F(2,75)=1.76$ & $F(2,341)=9.06^{* * *}$ \\
\hline & Block & $F(1,2715)=19.24 * * *$ & $F(1,375)=7.94 * *$ & $F(1,1705)=18.46^{* * *}$ \\
\hline & Pose by Belief & $F(4,2715)=48.95 * * *$ & $F(4,375)=4.74 * * *$ & $F(4,1705)=20.47 * * *$ \\
\hline & Pose by Block & $F(2,2715)=1.16$ & $F(2,375)=2.02$ & $F(2,1705)=1.26$ \\
\hline & Belief by Block & $F(2,2715)=0.3$ & $F(2,375)=2.9$ & $F(2,1705)=0.21$ \\
\hline & Pose by Belief by Block & $F(4,2715)=1.02$ & $F(4,375)=0.34$ & $F(4,1705)=0.28$ \\
\hline \multirow[t]{7}{*}{ Anger } & Pose & $F(2,2715)=103.74 * * *$ & $F(2,375)=51.67 * * *$ & $F(2,1705)=255.28 * * *$ \\
\hline & Belief & $F(2,543)=6.39 * *$ & $F(2,75)=0.03$ & $F(2,341)=1.23$ \\
\hline & Block & $F(1,2715)=2.96$ & $F(1,375)=0.57$ & $F(1,1705)=1.12$ \\
\hline & Pose by Belief & $F(4,2715)=26.8 * * *$ & $F(4,375)=2.73^{*}$ & $F(4,1705)=10.04 * * *$ \\
\hline & Pose by Block & $F(2,2715)=0.29$ & $F(2,375)=0.26$ & $F(2,1705)=0.12$ \\
\hline & Belief by Block & $F(2,2715)=0.48$ & $F(2,375)=0.13$ & $F(2,1705)=0.17$ \\
\hline & Pose by Belief by Block & $F(4,2715)=0.74$ & $F(4,375)=0.5$ & $F(4,1705)=0.3$ \\
\hline
\end{tabular}


Table S3.

Study 1-3 mean differences in self-reported happiness and anger after posing happy vs. neutral vs. angry facial expressions in the positive-hypothesis, nohypothesis, and nil-hypothesis conditions. Study 1, 2, and 3 pairwise comparison tests had 1235, 945, 645, degrees of freedom respectively.

\begin{tabular}{|c|c|c|c|c|c|c|c|c|}
\hline \multirow[b]{2}{*}{ Study } & \multirow[b]{2}{*}{ Emotion report } & \multirow[b]{2}{*}{ Pose contrast } & \multicolumn{2}{|c|}{ Positive-hypothesis } & \multicolumn{2}{|c|}{ No-hypothesis } & \multicolumn{2}{|c|}{ Nil-hypothesis } \\
\hline & & & $t$ & $95 \% \mathrm{CI}$ & $t$ & $95 \% \mathrm{CI}$ & $t$ & $95 \% \mathrm{CI}$ \\
\hline \multirow[t]{6}{*}{ Study 1} & Happiness & Happy - Neutral & $16.33 * * *$ & {$[1.12,1.43]$} & $10.82 * * *$ & {$[0.73,1.05]$} & $4.25 * * *$ & {$[0.18,0.48]$} \\
\hline & & Happy - Anger & $17.5^{* * *}$ & {$[1.21,1.52]$} & $11.77 * * *$ & {$[0.81,1.13]$} & $5.54 * * *$ & {$[0.28,0.58]$} \\
\hline & & Neutral - Anger & 1.17 & {$[-0.06,0.24]$} & 0.95 & {$[-0.08,0.24]$} & 1.29 & {$[-0.05,0.25]$} \\
\hline & Anger & Happy - Neutral & $-2.58 * *$ & {$[-0.26,-0.04]$} & 0.25 & {$[-0.1,0.13]$} & 1.15 & {$[-0.05,0.18]$} \\
\hline & & Happy - Anger & $-14.34 * * *$ & {$[-0.93,-0.71]$} & $-8.91 * * *$ & {$[-0.66,-0.42]$} & $-2.94 * * *$ & {$[-0.28,-0.06]$} \\
\hline & & Neutral - Anger & $-11.76^{* * *}$ & {$[-0.78,-0.56]$} & $-9.16^{* * *}$ & {$[-0.67,-0.43]$} & $-4.09 * * *$ & {$[-0.34,-0.12]$} \\
\hline \multirow[t]{6}{*}{ Study 2} & Happiness & Happy - Neutral & $10.36 * * *$ & {$[0.89,1.31]$} & $7.62 * * *$ & {$[0.61,1.03]$} & $6.84 * * *$ & {$[0.55,0.99]$} \\
\hline & & Happy - Anger & $13.22 * * *$ & {$[1.19,1.61]$} & $10.14 * * *$ & {$[0.88,1.3]$} & $7.83 * * *$ & {$[0.66,1.1]$} \\
\hline & & Neutral - Anger & $2.86^{* * *}$ & {$[0.1,0.51]$} & $2.52 * *$ & {$[0.06,0.48]$} & 0.99 & {$[-0.11,0.33]$} \\
\hline & Anger & Happy - Neutral & -0.88 & {$[-0.28,0.11]$} & -0.05 & {$[-0.2,0.19]$} & -0.37 & {$[-0.24,0.17]$} \\
\hline & & Happy - Anger & $-9.09 * * *$ & {$[-1.09,-0.7]$} & $-8.13 * * *$ & {$[-1.01,-0.62]$} & $-5.64 * * *$ & {$[-0.79,-0.38]$} \\
\hline & & Neutral - Anger & $-8.2 * * *$ & {$[-1,-0.62]$} & $-8.07 * * *$ & {$[-1.01,-0.61]$} & $-5.27 * * *$ & {$[-0.75,-0.35]$} \\
\hline \multirow[t]{6}{*}{ Study 3} & Happiness & Happy - Neutral & $8.37 * * *$ & {$[0.79,1.28]$} & & & $4.53 * * *$ & {$[0.3,0.76]$} \\
\hline & & Happy - Anger & $10.55 * * *$ & {$[1.06,1.55]$} & & & $6.76^{* * *}$ & {$[0.56,1.02]$} \\
\hline & & Neutral - Anger & $2.17 *$ & {$[0.03,0.51]$} & & & $2.23 *$ & {$[0.03,0.49]$} \\
\hline & Anger & Happy - Neutral & 0.18 & {$[-0.21,0.26]$} & & & 1.11 & {$[-0.1,0.35]$} \\
\hline & & Happy - Anger & $-11.21 * * *$ & {$[-1.57,-1.1]$} & & & $-5.67 * * *$ & {$[-0.86,-0.42]$} \\
\hline & & Neutral - Anger & $-11.4 * * *$ & {$[-1.59,-1.12]$} & & & $-6.78 * * *$ & {$[-0.99,-0.54]$} \\
\hline
\end{tabular}

$* p<.05 ; * * p<.01 ; * * * p<.001$ 
Table S4.

Study 4-6 mean differences in self-reported happiness and anger after posing happy vs. neutral vs. angry facial expressions for participants who either believed, were unsure, or did not believe in facial feedback effects. Study 4-6 pairwise comparisons had 2715, 375, 1705, degrees of freedom respectively.

\begin{tabular}{|c|c|c|c|c|c|c|c|c|}
\hline \multirow[b]{2}{*}{ Study } & \multirow[b]{2}{*}{ Emotion report } & \multirow[b]{2}{*}{ Pose contrast } & \multicolumn{2}{|c|}{ Believer } & \multicolumn{2}{|c|}{ Unsure } & \multicolumn{2}{|c|}{ Nonbeliever } \\
\hline & & & $t$ & $95 \% \mathrm{CI}$ & $t$ & $95 \% \mathrm{CI}$ & $t$ & $95 \% \mathrm{CI}$ \\
\hline \multirow[t]{6}{*}{ Study 4} & Happiness & Happy - Neutral & $25.62 * * *$ & {$[1.05,1.22]$} & $5.64 * * *$ & {$[0.43,0.9]$} & $3.29 * * *$ & {$[0.09,0.35]$} \\
\hline & & Happy - Anger & $30.49 * * *$ & {$[1.26,1.43]$} & $6.4 * * *$ & {$[0.52,0.99]$} & $5.56 * * *$ & {$[0.24,0.5]$} \\
\hline & & Neutral - Anger & $4.87 * * *$ & {$[0.13,0.3]$} & 0.76 & {$[-0.14,0.32]$} & $2.26^{*}$ & {$[0.02,0.28]$} \\
\hline & Anger & Happy - Neutral & $-2.48 * *$ & {$[-0.18,-0.02]$} & 0.1 & {$[-0.2,0.22]$} & $2.8 * *$ & {$[0.05,0.29]$} \\
\hline & & Happy - Anger & $-23.54 * * *$ & {$[-1.02,-0.86]$} & $-3.56 * * *$ & {$[-0.59,-0.17]$} & $-4.1 * * *$ & {$[-0.36,-0.13]$} \\
\hline & & Neutral - Anger & $-21.07 * * *$ & {$[-0.92,-0.77]$} & $-3.66 * * *$ & {$[-0.6,-0.18]$} & $-6.9 * * *$ & {$[-0.53,-0.3]$} \\
\hline \multirow[t]{6}{*}{ Study 5} & Happiness & Happy - Neutral & $9.65 * * *$ & {$[0.9,1.36]$} & $5.84 * * *$ & {$[0.56,1.13]$} & $2.87 * * *$ & {$[0.13,0.7]$} \\
\hline & & Happy - Anger & $11.2 * * *$ & {$[1.08,1.54]$} & $5.9 * * *$ & {$[0.57,1.14]$} & $4.9 * * *$ & {$[0.43,1]$} \\
\hline & & Neutral - Anger & 1.55 & {$[-0.05,0.41]$} & 0.05 & {$[-0.28,0.29]$} & $2.03 *$ & {$[0.01,0.58]$} \\
\hline & Anger & Happy - Neutral & $-2.67 * *$ & {$[-0.71,-0.11]$} & -0.25 & {$[-0.26,0.2]$} & -1.51 & {$[-0.48,0.06]$} \\
\hline & & Happy - Anger & $-6.55 * * *$ & {$[-1.3,-0.7]$} & $-3.74 * * *$ & {$[-0.68,-0.21]$} & $-6.49 * * *$ & {$[-1.16,-0.62]$} \\
\hline & & Neutral - Anger & $-3.88 * * *$ & {$[-0.89,-0.29]$} & $-3.48 * * *$ & {$[-0.65,-0.18]$} & $-4.98 * * *$ & {$[-0.95,-0.41]$} \\
\hline \multirow[t]{6}{*}{ Study 6} & Happiness & Happy - Neutral & $15.79 * * *$ & {$[1.06,1.36]$} & $12.96 * * *$ & {$[0.73,0.99]$} & $2.12 *$ & {$[0.02,0.4]$} \\
\hline & & Happy - Anger & $18.03 * * *$ & {$[1.23,1.53]$} & $16 * * *$ & {$[0.93,1.19]$} & $4.26 * * *$ & {$[0.23,0.62]$} \\
\hline & & Neutral - Anger & $2.23 *$ & {$[0.02,0.32]$} & $3.04 * * *$ & {$[0.07,0.33]$} & $2.14 *$ & {$[0.02,0.41]$} \\
\hline & Anger & Happy - Neutral & 0.42 & {$[-0.14,0.21]$} & $4.34 * * *$ & {$[0.15,0.39]$} & $2.6^{* *}$ & {$[0.04,0.3]$} \\
\hline & & Happy - Anger & $-12.59 * * *$ & {$[-1.28,-0.94]$} & $-10.07 * * *$ & {$[-0.74,-0.5]$} & $-7.07 * * *$ & {$[-0.6,-0.34]$} \\
\hline & & Neutral - Anger & $-13.01 * * *$ & {$[-1.32,-0.97]$} & $-14.41 * * *$ & {$[-1.01,-0.77]$} & $-9.67 * * *$ & {$[-0.77,-0.51]$} \\
\hline
\end{tabular}

$* p<.05 ; * * p<.01 ; * * * p<.001$ 
FACIAL FEEDBACK AND STUDY ARTIFACTS

\section{Exploratory between-subject test of facial feedback effects}

In Studies 1-6, we manipulated Pose within-subjects. However, some readers may wonder if similar patterns would have been obtained if we had manipulated Pose between-subjects. To examine this, we performed exploratory between-subjects analyses using data pooled from Studies 1-3. Given that participants completed the facial posing tasks in random order, we were able to compare emotion responses between (1) the first trial from participants who completed a happy pose first, (2) the first trial from participants who completed a neutral pose first, and (3) the first trial from participants who completed an angry pose first. In other words, by focusing on participants' first facial pose trials, we are able to examine data from a 3(Pose: happy, neutral, angry) x 3(Demand: positive-hypothesis, no-hypothesis, nil-hypothesis) between-subject design.

Results from the between-subject analyses confirmed that observed facial feedback effects are not fully driven by demand characteristics. We modeled participants' emotion reports using linear regression with Pose and Demand entered as effects-coded factors, their higher-order interaction, and Type III Sums of Squares. For self-reported happiness, results confirmed that (a) the effect of Pose was moderated by Demand, F(4, 564) = 4.57, $\mathrm{p}<.002$ ), and (b) the effects of Pose was significant in all levels of Demand (p's $<.0001)$. For selfreported anger, results provided marginally significant evidence that the effect of Pose was moderated by Demand, $\mathrm{F}(4,564)=1.96, \mathrm{p}=.10$. Most importantly, though, the effect of Pose on self-reported anger was significant in all levels of Demand (p's $<.0002)$.

Unfortunately, we did not record order data for Studies 5-6 and could not perform similar betweensubjects analyses on the moderating role of participants beliefs about facial feedback. However, given that within- and between-subjects analyses yielded similar conclusions about the moderating role of demand characteristics, we suspect they would also yield similar conclusions about the moderating role of participants beliefs about facial feedback.

\section{Are facial feedback effects emotion- or valence-specific?}

There is an ongoing debate in the affective sciences regarding whether emotions are best conceptualized as discrete categories (e.g., anger and sadness) or as phenomena that are reducible to simpler dimensions, such 
as valence (i.e., the degree of positivity and negativity) and arousal. Similarly, researchers have debated whether facial feedback has discrete emotion-specific vs. valence-specific effects on emotional experience (for a review, see Coles, Larsen, \& Lench, 2019).

When designing the present set of studies, we felt that it was important to first thoroughly address concerns about study artifacts before dedicating resources to understanding facial feedback characteristics and mechanisms. Our studies were not specifically designed to answer questions about the specificity of facial feedback effects. (E.g., we did not include multiple same-valence facial feedback manipulations; we did not measure generally positivity/negativity; etc.) Nevertheless, our data to allow us to weigh in on this debate in an exploratory manner.

If facial feedback effects are valence-specific, we should expect to observe two patterns of results. First, we should observe that emotion-specific poses decrease opposite-valence reports. (E.g., posing angry vs. neutral expressions should decrease happiness reports; posing happy vs. neutral expressions should decrease anger reports.) Second, we should observe that emotion-specific poses increase all same-valence emotion reports. (E.g., posing angry vs. neutral expressions should not only increase anger, but also other negative-valenced emotions such as fear.)

As shown in Tables S3 and S4, results provided inconsistent evidence for the first prediction. We sometimes observed that participants reported (a) less happiness after posing angry vs. neutral expressions, and (b) less anger after posing happy vs. neutral expressions. As shown in Table S5, we also sometimes observed that (c) participants reported less fear after posing happy vs. neutral expressions, an emotion report that we initially used as a filler set of items. However, these patterns were not consistently observed across levels of the demand characteristics manipulations, levels of participants' beliefs, and studies.

As shown in Table S5, exploratory analyses also provided non-robust evidence for the second prediction. Consistent with the notion that facial feedback effects are valence-specific, we sometimes observed that participants reported higher levels of fear after posing angry vs. neutral expressions. The pattern of results was numerically consistent across all studies, but the statistical significance of the results were not consistent. 
Taken together, we suggest that our data do not reliably favor either emotion-specific or valence-specific facial feedback accounts. 


\section{Table S5.}

Study 1-6 mean differences in self-reported fear after posing happy vs. neutral vs. angry facial expressions. Study 1-6 pairwise comparison tests had 1235, 945, 645, 2715, 375, and 1705 degrees of freedom respectively.

\begin{tabular}{llll}
\hline Study & Pose contrast & $\boldsymbol{t}$ & $\mathbf{9 5 \%}$ CI \\
\hline Study 1 & Happy - Neutral & -0.28 & {$[-0.02,0.02]$} \\
& Happy - Anger & -1.67 & {$[-0.04,0]$} \\
Neutral - Anger & -1.39 & {$[-0.03,0.01]$} \\
Study 2 & Happy - Neutral & -0.44 & {$[-0.05,0.03]$} \\
& Happy - Anger & $-3.02^{* * *}$ & {$[-0.1,-0.02]$} \\
& Neutral - Anger & $-2.58^{* *}$ & {$[-0.09,-0.01]$} \\
Study 3 & Happy - Neutral & $-3.55 * * *$ & {$[-0.25,-0.07]$} \\
& Happy - Anger & $-3.99 * * *$ & {$[-0.27,-0.09]$} \\
Study 4 & Neutral - Anger & -0.43 & {$[-0.11,0.07]$} \\
& Happy - Neutral & -0.37 & {$[-0.04,0.03]$} \\
& Happy - Anger & $-2.04 *$ & {$[-0.07,0]$} \\
Study 5 & Neutral - Anger & -1.67 & {$[-0.07,0.01]$} \\
& Happy - Neutral & -1.14 & {$[-0.08,0.02]$} \\
& Happy - Anger & -1.19 & {$[-0.08,0.02]$} \\
& Neutral - Anger & -0.05 & {$[-0.05,0.05]$} \\
& Happy - Neutral & -1.24 & {$[-0.07,0.02]$} \\
& Happy - Anger & $-5.63 * * *$ & {$[-0.17,-0.08]$} \\
& Neutral - Anger & $-4.39 * * *$ & {$[-0.14,-0.05]$} \\
\hline
\end{tabular}

${ }^{*} p<.05 ; * * p<.01 ; * * * p<.001$ 
FACIAL FEEDBACK AND STUDY ARTIFACTS

\section{Study 1, 2, and 5 Ethnicity Data}

We did not measure participants' ethnicity in our studies. Nevertheless, for the studies conducted in the United States, we were able to recover data regarding the ethnicity of the participant pool population that we sampled from. See Table S6 for more information.

\section{Table S6.}

Participant pool ethnicity data for Studies 1, 2, and 5.

\begin{tabular}{llll}
\hline Ethnicity & Study 1 & Study 2 & Study 5 \\
\hline Non-Hispanic European American / White & $77.48 \%$ & $75.93 \%$ & $75.81 \%$ \\
African American / Black & $6.56 \%$ & $8.72 \%$ & $7.23 \%$ \\
Asian / Asian American / Pacific Islander & $5.25 \%$ & $4.68 \%$ & $4.13 \%$ \\
Hispanic / Latino/a & $3.37 \%$ & $2.8 \%$ & $4.13 \%$ \\
Native American / American Indian & $0.31 \%$ & $0.2 \%$ & $0.59 \%$ \\
Bi- or Multi-Racial & $3.76 \%$ & $4.03 \%$ & $4.13 \%$ \\
Other & $2.36 \%$ & $2.54 \%$ & $2.06 \%$ \\
Declined to Answer & $0.92 \%$ & $1.11 \%$ & $1.92 \%$ \\
\hline
\end{tabular}

\section{Belief as a Continuous Variable}

In Study 4, we measured the extent to which each participant believed that posing facial expressions could influence emotion using a continuous measure ( $0=$ strong disbelief; $4=$ strong belief). Based on these ratings, we categorized participants based on whether they believed ( 4 or 3 on the aforementioned scale; $n=$ $344 ; 63 \%$ of sample), were unsure if ( 2 on the aforementioned scale; $n=48 ; 9 \%$ of sample), or did not believe ( 1 or 0 on the aforementioned scale; $n=154 ; 28 \%$ of sample) posing facial expressions can influence emotions. An anonymous reviewer pointed out that there are several limitations to trichotomizing a continuous variable (Altman \& Royston, 2006). Here, we describe the similar results obtained when belief is modeled as a continuous variable.

\section{The Effects of Demand Characteristics on Participants' Beliefs in Facial Feedback Effects}

Results from a one-way ANOVA indicated that ratings of participants' belief about facial feedback effects differed across the three Demand conditions, $F(2,543)=23.16, p<.001$. Participants were slightly more convinced that facial feedback influences emotion in the positive expectation $(M=2.80, S D=1.18)$ vs. control 
$(M=2.58, S D=1.25), t(543)=1.55, p=.12, M_{\text {diff }} 95 \%$ CI $[-0.06,0.51]$, and null expectation condition $(M=$ $1.96, S D=1.45), t(543)=6.64, p<.001, M_{\text {diff }} 95 \%$ CI $[0.60,1.10]$. Participants were also more convinced that facial feedback influences emotion in the control vs. null expectation condition, $t(543)=4.24, p<.001, M_{\text {diff }}$ 95\% CI $[0.33,0.91]$. These results indicate that the hypothesis communicated to participants impacted their beliefs about facial feedback.

\section{The Moderating Role of Participants’ Beliefs in Facial Feedback Effects}

To test the moderating role of participants' beliefs about facial feedback, we modeled self-reported happiness and anger using linear mixed-effects regression with Pose and Block entered as effect-coded factors, Belief entered mean-centered, all higher-order interactions, random-intercepts for each participant, and Type III Sums of Squares. $F$-values are from ANOVA tables with Satterthwaite degrees of freedom. For both selfreported happiness and anger, there were significant Pose by Belief interactions: happiness, $F(2,2720)=$ $123.35, p<.001$; anger, $F(2,2720)=66.22, p<.001$. Follow-up pairwise comparisons indicated that, regardless of their beliefs about facial feedback effects, participants reported (a) more happiness after posing happy vs. neutral and angry expressions, and (b) more anger after posing angry vs. neutral and happy expressions (Figure S2). 
Figure S2.

Self-reported happiness and anger for Study 1-3 participants who posed happy, neutral, and angry expressions. Data visualized separately for each level of belief in facial feedback effects $(0=$ strong disbelief; $4=$ strong belief).
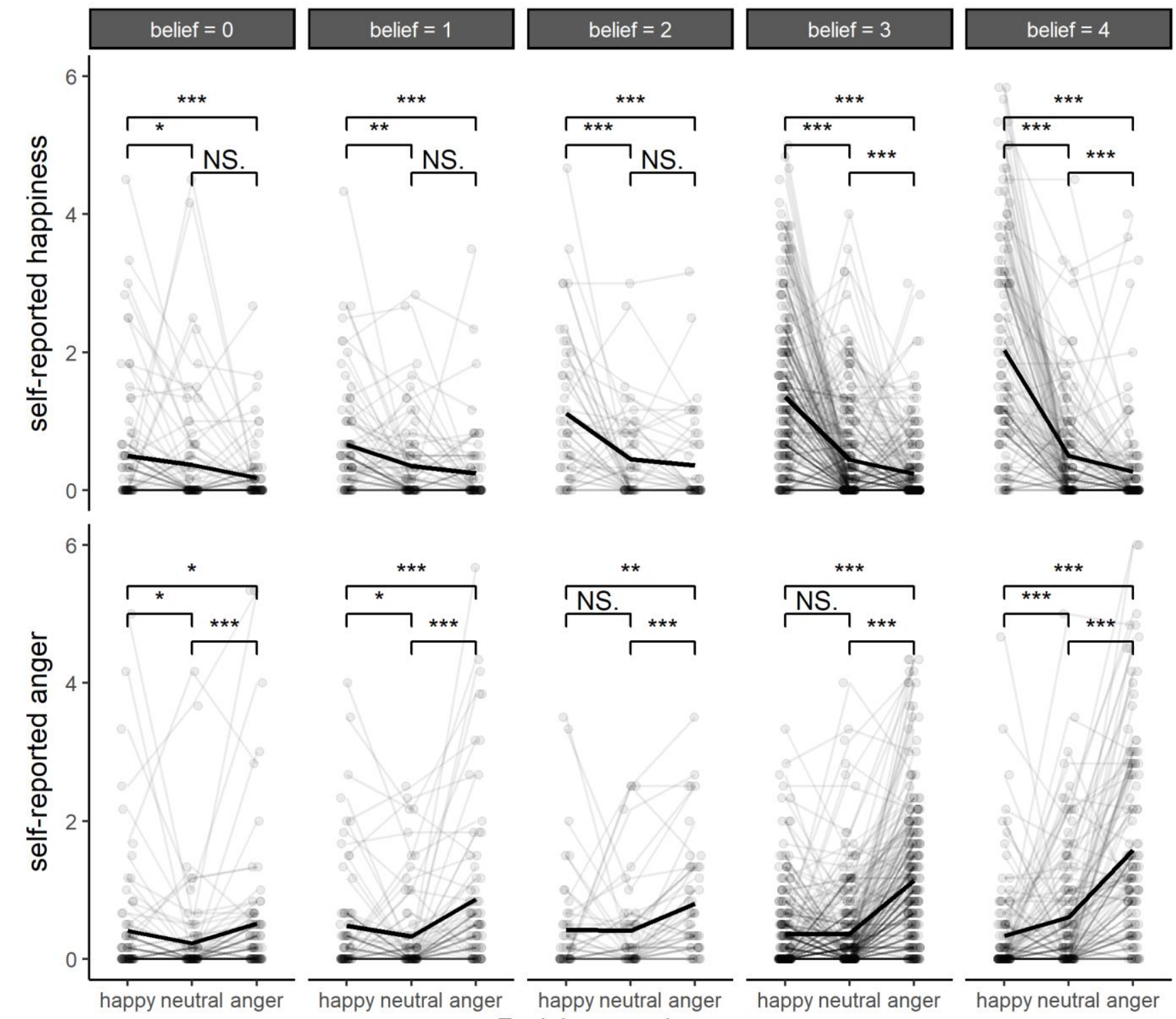

Facial expression pose

Note. Grey points represent jittered participant-level observations, bolded black circles represent group-level means, and bolded black vertical lines represent group-level standard errors. Wilcoxon tests were used for all pairwise comparison tests.

$* p<.05 ; * * p<.01 ; * * * p<.001$ 